\title{
Global Asymptotic Stability for a Periodic Delay Hematopoiesis Model with Impulses
}

\author{
Teresa Faria \\ Departamento de Matemática and CMAF-CIO, Faculdade de Ciências, \\ Universidade de Lisboa, Campo Grande, 1749-016 Lisboa, Portugal \\ e-mail: teresa.faria@fc.ul.pt \\ José J. Oliveira ${ }^{1}$ \\ CMAT and Departamento de Matemática e Aplicações, Escola de Ciências, \\ Universidade do Minho, Campus de Gualtar, 4710-057 Braga, Portugal \\ e-mail: jjoliveira@math.uminho.pt
}

\begin{abstract}
In this paper, sufficient conditions for the global asymptotic stability of a broad family of periodic impulsive scalar delay differential equations are obtained. These conditions are applied to a periodic hematopoiesis model with multiple time-dependent delays and linear impulses, in order to establish criteria for the global asymptotic stability of a positive periodic solution. The present results are discussed within the context of recent literature. In conclusion, when compared with previous works, not only sharper stability criteria are obtained here, even for models without impulses, but also the usual constraints imposed on the linear impulses are relaxed.
\end{abstract}

Keywords: hematopoiesis model, Mackey-Glass model, impulses, periodic solution, global asymptotic stability.

2010 Mathematics Subject Classification: 34K13, 34K45, 34K25, 92C50.

\section{Introduction}

Consider the family of scalar periodic delay differential equations (DDEs) with impulses given by

$$
\left\{\begin{array}{l}
x^{\prime}(t)+a(t) x(t)=\sum_{i=1}^{m} \beta_{i}(t) g_{i}\left(t, x\left(t-\tau_{i}(t)\right)\right), 0 \leq t \neq t_{k}, \\
x\left(t_{k}^{+}\right)-x\left(t_{k}\right)=b_{k} x\left(t_{k}\right), \quad k \in \mathbb{N}
\end{array}\right.
$$

where the coefficient functions $a(t), \beta_{i}(t), g_{i}(t, x)$ and the delay functions $\tau_{i}(t) \geq 0(1 \leq i \leq$ $m)$ are continuous and periodic in $t$ with a common period $\omega>0, b_{k}$ are constants and the sequence of impulses at instants $t_{k}(k \in \mathbb{N})$ occur with periodicity $\omega$.

For impulsive DDEs of the form (1.1), in [1] the authors gave very general conditions for the existence of a positive periodic solution - which need not be unique. The study in [1] was mostly motivated by the fact that the class of impulsive DDEs (1.1) includes a number of important models used in mathematical biology. As we shall see, this biological framework imposes some natural additional conditions on the coefficient functions and impulses in (1.1), as well as restricts the set of admissible solutions to the set of nonnegative functions. Of course, when the results in [1] guarantee that a positive periodic solution must exist, a topic

\footnotetext{
${ }^{1}$ Corresponding author.
} 
of major importance is the convergence to this periodic solution (as $t \rightarrow \infty$ ) of all positive solutions.

In this paper, we investigate the asymptotic behaviour of solutions of the following delayed periodic hematopoiesis model with linear impulses and multiple time-dependent delays:

$$
\left\{\begin{array}{l}
y^{\prime}(t)+a(t) y(t)=\sum_{i=1}^{m} \frac{\beta_{i}(t)}{1+y\left(t-\tau_{i}(t)\right)^{n}}, 0 \leq t \neq t_{k}, \\
y\left(t_{k}^{+}\right)-y\left(t_{k}\right)=b_{k} y\left(t_{k}\right), \quad k \in \mathbb{N},
\end{array}\right.
$$

where $m \in \mathbb{N}, n \in(0, \infty),\left(t_{k}\right)_{k \in \mathbb{N}}$ and $\left(b_{k}\right)_{k \in \mathbb{N}}$ are $\omega$-periodic real sequences, and $a, \beta_{i}: \mathbb{R} \rightarrow$ $[0, \infty), \tau_{i}: \mathbb{R} \rightarrow[0, \infty)$ are continuous and $\omega$-periodic, for $i=1, \ldots, m$. Our main goal is to establish criteria for the global asymptotic stability of a positive periodic solution of (1.2).

This system generalizes one of the celebrated hematopoiesis models proposed by Mackey and Glass, and is embedded within the framework set by (1.1).

Hematopoiesis is the process of production, multiplication, regulation and specialization of blood cells in the bone marrow, until they become mature blood cells for release in the circulation bloodstream. Blood is a connective tissue which is made up of cellular elements - red blood cells, white blood cells and platelets - and an extracellular fluid, the plasma. Hematopoiesis begins with the differentiation and division of the multipotent hematopoietic stem cells: some of them remain hematopoietic stem cells, allowing hematopoiesis to continue, and others divide into lymphoid and myeloid stem cells; after several intermediate stages, the lymphoid stem cells give rise to the lymphocytes (various types of white blood cells), whereas the myeloid stem cells originate thrombocytes (platelets), erythrocytes (red blood cells), and other white blood cells: basophils, neutrophils, eosinophils and monocytes. The stem cells take time to multiply and specialize into mature blood cells, thus time lags occur. Typically the plasma is replaced within 24 hours, but it takes between days and several weeks to replace the different types of blood cells. For instance, neutrophils mature in about 2 weeks while the basophils mature in 7 days.

In 1977, Mackey and Glass [2] introduced scalar differential equations with a time delay to describe some physiological mechanisms, such as the hematopoiesis process. Two type of models of the form $x^{\prime}(t)=-\gamma x(t)+f(x(t-\tau))$ were proposed in [2], a first one where the production of cells is given by a monotone decreasing function $f(x)=\beta_{0} \frac{\theta^{n}}{\theta^{n}+x^{n}}(n>0)$, and a second one with a unimodal production function $f(x)=\beta_{0} \frac{x}{\theta^{n}+x^{n}}(n>1)$, where all the constants are positive. We remark that Hill functions $f(x)=\beta_{0} \frac{\theta^{n}}{\theta^{n}+x^{n}}$ are often used as production or growth functions in physiological modelling or population dynamics, where $\beta_{0}$ is an intrinsic rate and the coefficients $\theta, n$ are determined so that $f$ fits well with clinical and experimental data $[3,4]$. After normalization of the coefficients, the mathematical models proposed by Mackey and Glass [2] with decreasing and unimodal production rate are given by, respectively,

$$
y^{\prime}(t)+\gamma y(t)=\frac{\beta}{1+y(t-\tau)^{n}}, \quad t \geq 0
$$

and

$$
y^{\prime}(t)+\gamma y(t)=\frac{\beta y(t-\tau)}{1+y(t-\tau)^{n}}, \quad t \geq 0
$$

with $\gamma, \beta>0$ and $n>0$ in (1.3) and $n>1$ in (1.4). In [5, 6], Mackey used (1.3),(1.4) as appropriate models to reproduce hematopoiesis. In (1.3), $y(t)$ is the density of mature cells 
in the circulation, $\tau$ is the time delay between the production of immature cells in the bone marrow and their maturation for release in the blood, $\gamma$ is a destruction rate and $\beta$ is the maximal production rate. The stability of the unique positive equilibrium point of (1.3) has been studied by several authors (see e.g. [7, 8, 9, 10, 11]).

For the last decades, a large number of generalizations of the hematopoiesis MackeyGlass equations has been suggested and analysed. For some detail about the biological mechanisms of hematopoiesis, a review on hematological disorders and derivation of several mathematical models, see the works of Bélair et al. [12], Mahaffy et al. [4], Haurie et al. [13], Adimy et al. [14], Langlois et al. [15], and references therein. Namely, in [12] the authors derived and studied a model for erythropoiesis (the process of production of red blood cells) consisting of a system of two autonomous DDEs, one for the level of erythropoietin (the hormone that stimulates reproduction of erythrocytes) and the other for the population of mature erythrocytes, with two different delays: a first delay of 6-7 days for the time red blood cells take to mature in the bone marrow, and a second delay of 120 days for the lifespan of the mature cells in the bloodstream. As shown by the authors, their model fits well with the experimental data referring to normal erythropoiesis, but not so well with observed hematological disorders. More recently, in [15] again a system of autonomous DDEs with two delays was deduced as a model for the normal and pathological dynamics of blood platelets, and careful estimations of the parameters according to laboratory and clinical data performed. In both $[12,15]$, the local stability of the unique positive equilibrium and occurence of Hopf bifurcation were analysed.

It is however natural to claim that more realistic models are obtained with nonautonomous DDEs, where the parameters are not fixed constants. Particularly important are models with periodic coefficients and delays, since they allow us to take into account the periodicity of the environment, which plays an important role in many biological and ecological dynamical systems. In what concerns the blood cells in circulation, there are several clinical experiments noting that its density undergoes through seasonal fluctuations, due to temperature, weather and food supply, see [16] and references therein. Some results on existence and/or attractivity of positive periodic solutions for periodic versions of (1.3) can be found in $[1,17,18,19,20]$. See also $[16,21,22]$, for more results, surveys on periodic hematopoiesis delayed models of the Mackey-Glass type, as well as further references. On the other hand, some evolutionary systems go through abrupt changes, due to predictable or sudden external phenomena, such as radiation, drug administration or other forms of stress in the case of blood cells, or weather, resource availability, or mating habits in population dynamics processes. These phenomena are better described by the so-called impulsive differential equations. For a discussion of the role of impulses, see e.g. [23, 24, 25]. The modification of (1.3) according to a periodic environmental variation and subject to periodic linear impulses reads as

$$
\left\{\begin{array}{l}
y^{\prime}(t)+a(t) y(t)=\frac{\beta(t)}{1+y(t-\tau(t))^{n}}, 0 \leq t \neq t_{k} \\
y\left(t_{k}^{+}\right)-y\left(t_{k}\right)=b_{k} y\left(t_{k}\right), \quad k \in \mathbb{N}
\end{array}\right.
$$

where $n \in(0, \infty)$ and $a, \beta, \tau: \mathbb{R}^{+} \rightarrow \mathbb{R}^{+}$are periodic with a common period $\omega>0$, and the sequences of the instants of impulses $\left(t_{k}\right)$ and impulsive coefficients $\left(b_{k}\right)$ are $\omega$-periodic.

As shown in the aforementioned papers $[12,15]$, DDEs with two or more different delays appear naturally in hematological models, as well as in other real-world physiological phenomena; see [26] for an explanation of this situation in gene regulatory systems and 
stability results for a unimodal non-autonomous Mackey-Glass equation with two delays. Since different blood cells take different times to mature in the bone marrow, production functions with different time lags should be taken into consideration [16]. Moreover, from a mathematical point of view, the treatment of the impulsive model (1.2) with several delays, rather than a single delay as in (1.5), is more interesting and embracing of other processes.

We should mention that many authors have remarked that in real world applications almost periodic effects are more frequent than periodic ones, thus almost periodic variants of the models under consideration have been introduced. Recently, there has been a number of studies concerning the existence of a positive almost periodic solution for almost periodic hematopoiesis models $[27,28]$, however few authors have investigated almost periodic Mackey-Glass-type models with impulses [29, 30]. Although not explored here, in fact our technique can be used to study the global attractivity of a positive almost periodic solution for almost periodic models (1.2), provided that such a solution exists. See further comments in the last section of the paper.

In spite of the extensive number of works dedicated to DDEs of the Mackey-Glass-type and their generalizations, as well as on alternative models of hematopoiesis, the literature on periodic impulsive versions of (1.3) is almost non-existent. It is worthwhile to emphasize the work of Sacker and Alzabut [24], where the particular case of system (1.5) with one constant delay multiple of the period (i.e., $\tau(t) \equiv q \omega$ for some $q \in \mathbb{N}$ ) and $n \in\{1,2, \ldots\}$ was studied, and sufficient conditions for the global attractivity of a unique positive periodic solution derived. The model (1.2) without impulses was studied by Liu et. al. [18], where the existence of a positive $\omega$-periodic solution and its global attractivity (in the set of all positive solutions) were guaranteed under some sufficient conditions, which differ for each one of the situations $0<n \leq 1$ and $n>1$.

On the other hand, in $[31,32]$ the authors of this paper analysed the global asymptotic stability of the zero solution for a very general class of scalar delay differential equations with impulses. In this paper, the positive periodic solution $y^{*}(t)$ of $(1.2)$, whose existence follows under some conditions in [1], is first translated to the origin and, inspired by the conclusions drawn in $[31,32]$, new criteria for its global attractivity are obtained.

As in [18, 21], here the cases $0<n \leq 1$ and $n>1$ are studied separately: note that the behaviour of the nonlinearity is quite different in each of these two situations, therefore it is natural to treat them separately. To make this difference clearer, we recall that for the non-impulsive autonomous version of (1.2),

$$
y^{\prime}(t)+a y(t)=\sum_{i=1}^{m} \frac{\beta_{i}}{1+y\left(t-\tau_{i}\right)^{n}} \quad\left(a, \beta_{i}, \tau_{i}, n>0\right),
$$

the global asymptotic stability of the positive equilibrium $y^{*}$ has been proven for $0<n \leq 1$ without further constraints; whereas for $n>1$ several additional conditions have been established in order to guarantee its global attractivity $[11,21]$. For the periodic version of (1.6) with $n \in(0,1]$, it remains an open question whether the positive $\omega$-periodic solution is always a global attractor of all the positive solutions. The non-impulsive version of (1.2) with $0<n \leq 1$ was further explored in a recent paper [33], so in terms of examples we will focus here on several special cases of model (1.2) with $n>1$.

We now describe briefly the contents of the paper. In Section 2, we introduce some notation and recall some results on global attractivity, established by the authors in [31] for a family of scalar impulsive DDEs more general than (1.1). For the case of periodic DDEs with linear impulses, a stronger criterion is derived in Section 3. Section 4 is the core of 
the paper, where the stability of the positive periodic solution $y^{*}(t)$ of $(1.2)$ is addressed. It begins with some preparatory propositions, where uniform lower and upper bounds for $y^{*}(t)$ are established. Then, the attractivity of $y^{*}(t)$ of (1.2) is studied for both $0<n \leq 1$ and $n>1$ cases; special attention is given to hematopoiesis models (1.2) with delays which are multiple of the period. Several models and relevant comparison with results in recent literature are presented in Section 5. The paper ends with a short section of conclusions.

\section{Notations and preliminary results}

For model (1.2) under study, the following assumptions will be imposed:

(H1) the functions $a(t), \beta_{i}(t), \tau_{i}(t)$ are continuous, nonnegative and $\omega$-periodic (for some constant $\omega>0)$, with $a(t), \beta(t):=\sum_{i=1}^{m} \beta_{i}(t)$ not identically zero, $t \in \mathbb{R}, i=1, \ldots, m$;

(H2) there is $p \in \mathbb{N}$ such that $0<t_{1}<t_{2}<\cdots<t_{p}<\omega$ and

$$
t_{k+p}=t_{k}+\omega, b_{k+p}=b_{k}, \quad k \in \mathbb{N}
$$

(H3) the constants $b_{1}, \ldots, b_{p} \in \mathbb{R}$ satisfy $b_{k}>-1$;

(H4) $\prod_{k=1}^{p}\left(1+b_{k}\right)<\mathrm{e}^{\int_{0}^{\omega} a(t) d t}$.

As already mentioned, due to the biological interpretation of the model, only positive solutions of (1.2) should be considered, therefore a suitable set of nonnegative functions will be taken as the set of initial conditions. Note that hypothesis (H3) guarantees that solutions which are positive before the impulsive point $t_{k}$, i.e. with $y\left(t_{k}^{-}\right)=y\left(t_{k}\right)>0$, remain positive after the impulse at $t_{k}$, i.e. $y\left(t_{k}^{+}\right)>0, k \in \mathbb{N}$. Hypotheses (H1)-(H2) prescribe the $\omega$ periodic impulsive behaviour, whereas (H4) imposes that, on each interval of length $\omega$, the impulses cannot be too large when compared with the integral average of the nonnegative instantaneous coefficient $a(t)$ multiplied by the period $\omega$.

The assumptions imposed on the impulses are significantly weaker than the ones often found in the literature. In fact, instead of (H4), the strong constraint

$$
\prod_{k=1}^{p}\left(1+b_{k}\right)=1
$$

has been imposed; some authors have assumed that the function $\Theta(t):=\prod_{k: 0 \leq t_{k}<t}(1+$ $\left.b_{k}\right), t \geq 0$, is an $\omega$-periodic function [24]. However, as remarked by Liu and Takeuchi [23], if $\Theta(t)$ is $\omega$-periodic, then both (2.1) and (H2) hold. Moreover, when the impulses are all equal, $b_{k}=b$ for all $k \in \mathbb{N}$, then (2.1) is satisfied only if $b=0$, which means that there are no impulses.

Under hypotheses (H1)-(H4), Faria and Oliveira [1, Theorem 3.1] have recently shown that (1.2) has a positive $\omega$-periodic solution:

Theorem 2.1. [1] Under (H1)-(H4), there exists at least one positive w-periodic solution $y^{*}(t)$ of (1.2). 
We now introduce a family of scalar impulsive DDEs more general than (1.1). We start with an abstract setting to treat DDEs with impulses.

For a compact interval $[\alpha, \beta] \subset \mathbb{R}$, denote by $P C([\alpha, \beta] ; \mathbb{R})$ the space of bounded functions which are piecewise continuous on $[\alpha, \beta]$ and left continuous on $(\alpha, \beta]$, equipped with the supremum norm, $\|\varphi\|=\sup _{\theta \in[\alpha, \beta]}|\varphi(\theta)|$. Now, consider a finite set of bounded continuous delay functions $\tau_{i}:[0, \infty) \rightarrow[0, \infty), i=1, \ldots, m$, define

$$
\tau(t)=\max _{1 \leq i \leq m} \tau_{i}(t) \quad \text { and } \quad \bar{\tau}=\sup _{t \geq 0} \tau(t)
$$

and take the spaces $P C^{i}(t):=P C\left(\left[-\tau_{i}(t), 0\right] ; \mathbb{R}\right), P C(t):=P C([-\tau(t), 0] ; \mathbb{R})$ and $P C:=$ $P C([-\bar{\tau}, 0] ; \mathbb{R})$. For $x(t)$ defined on $[-\bar{\tau}, \alpha]$ and $0 \leq \sigma \leq \alpha$, we denote by $x_{\sigma}$ the function defined by $x_{\sigma}(s)=x(s+\sigma)$ for $s \in[-\tau(\sigma), 0]$ and by $x_{\sigma}^{i}$ the function defined by $x_{\sigma}^{i}(s)=$ $x(s+\sigma)$ for $s \in\left[-\tau_{i}(\sigma), 0\right], i=1, \ldots, m$.

Consider scalar impulsive delay differential equations of the form (see [31, 32])

$$
\left\{\begin{array}{l}
x^{\prime}(t)+a(t) x(t)=\sum_{i=1}^{m} f_{i}\left(t, x_{t}^{i}\right), 0 \leq t \neq t_{k}, \\
x\left(t_{k}^{+}\right)-x\left(t_{k}\right)=b_{k} x\left(t_{k}\right), \quad k \in \mathbb{N},
\end{array}\right.
$$

where: $x^{\prime}(t)$ is the left-hand derivative of $x(t) ; 0<t_{1}<t_{2}<\cdots<t_{k}<\cdots$ and $t_{k} \rightarrow \infty$; $a:[0, \infty) \rightarrow[0, \infty)$ is continuous with $\int_{0}^{\infty} a(t) d t=\infty ;\left(b_{k}\right)$ is a sequence of real numbers; for each $i \in\{1, \ldots, m\}, f_{i}$ is the restriction to $[0, \infty) \times P C^{i}(t)$ of a function $F_{i}:[0, \infty) \times P C \rightarrow \mathbb{R}$, in the sense that $F_{i}(t, \widetilde{\varphi}):=f_{i}\left(t, \widetilde{\varphi}_{\left.\right|_{\left[-\tau_{i}(t), 0\right]}}\right)=f_{i}(t, \varphi)$, for $t \geq 0$ and $\varphi \in P C^{i}(t)$, where by $\widetilde{\varphi} \in P C$ we mean the extension of $\varphi$ defined by $\varphi\left(-\tau_{i}(t)\right)$ on $\left[-\bar{\tau},-\tau_{i}(t)\right]$. In the sequel, we always suppose that either $F_{i}(t, \phi)$ is continuous in $(t, \phi)$, or is piecewise continuous in $(t, \phi)$ and continuous in the second variable. In these situations, we abuse the terminology and simply say either that $f_{i}$ is continuous, or that $f_{i}$ is piecewise continuous and continuous in $\varphi$. When uniqueness of solutions is required, we also impose that $F_{i}(t, \phi)$ is locally Lipschtiz continuous in the second variable. For more details, see [34, 35].

For the impulsive DDE (2.2), we consider initial conditions of the form $x_{t_{0}}=\varphi$, that is,

$$
x\left(t_{0}+s\right)=\varphi(s), \quad s \in\left[-\tau\left(t_{0}\right), t_{0}\right],
$$

with $t_{0} \geq 0$ and $\varphi \in P C\left(t_{0}\right)$. It is relevant to mention that these conditions together with the set of assumptions imposed below imply that the solution $x(t)=x\left(t, t_{0}, \varphi\right)$ of the initial value problem (2.2)-(2.3) exists and is defined on [ $\left.t_{0}, \infty\right)$, see e.g. [34].

As mentioned in the Introduction, the study of the global asymptotic stability of a positive periodic solution for the impulsive hematopoiesis model (1.2) is the major purpose of this paper. We first recall some usual definitions for stability.

Definition 2.1. Let $S \subseteq P C$ be a set of initial conditions, and suppose that 0 is an equilibrium of (2.2). The solution $x=0$ of $(2.2)$ is said to be stable in $S$ if for any $\varepsilon>0$ and $t_{0} \geq 0$, there exists $\delta=\delta\left(t_{0}, \varepsilon\right)>0$ such that

$$
\|\varphi\|<\delta \Rightarrow\left|x\left(t, t_{0}, \varphi\right)\right|<\varepsilon, \quad \text { for } t \geq t_{0}, \varphi \in S .
$$

We say that $x=0$ of (2.2) is globally attractive in $S$ if all solutions of (2.2) with initial conditions in $S$ tend to zero as $t \rightarrow \infty$. We say that $x=0$ is globally asymptotically stable (GAS) if it is stable and globally attractive. If it is clear which set $S$ we are dealing with, we may omit the reference to it. 
In previous papers [31,32], we studied the global asymptotic stability of the zero solution of impulsive DDEs (2.2) - in fact, more general impulses $\Delta x\left(t_{k}\right)=I_{k}\left(x\left(t_{k}\right)\right)$ given by continuous functions $I_{k}: \mathbb{R} \rightarrow \mathbb{R}, k=1,2, \ldots$, were considered in [31,32], but here we restrict our attention to the situation $I_{k}(u)=b_{k} u$. Therefore, we want to insert (1.2) in the framework provided by $(2.2)$.

First, we fix the set of admissible initial conditions. Due to its biological motivation, we only consider positive solutions, corresponding to initial conditions $y_{0}=\tilde{\varphi} \in P C_{0}^{+}$, where $\tilde{\varphi}(s)=\varphi(s)$ for $s \in[-\tau(0), 0]$ and $\tilde{\varphi}(s)=\varphi(-\tau(0))$ for $s \in[-\bar{\tau}, \tau(0)]$, and

$$
P C_{0}^{+}=\{\varphi \in P C: \varphi(\theta) \geq 0 \text { for } \theta \in[-\bar{\tau}, 0] \text { and } \varphi(0)>0\} .
$$

Even if not mentioned, only initial conditions in $\mathrm{PC}_{0}^{+}$are to be considered; henceforth, the stability and global attractivity of a solution of (1.2) will always refer to the set of initial conditions $P C_{0}^{+}$. Secondly, we translate an $\omega$-periodic solution $y^{*}(t)$ to the origin by the change of variables $x(t)=y(t)-y^{*}(t)$. System (1.2) is transformed into an impulsive DDE of the form (1.1), with each function $g_{i}:[0, \infty) \times \mathbb{R} \rightarrow \mathbb{R}(1 \leq i \leq m)$ defined by

$$
g_{i}(t, u)=\frac{1}{1+\left[u+y^{*}\left(t-\tau_{i}(t)\right)\right]^{n}}-\frac{1}{1+y^{*}\left(t-\tau_{i}(t)\right)^{n}},
$$

for $t \geq 0$ and $u \geq-y^{*}\left(t-\tau_{i}(t)\right)$, and e.g. $g_{i}(t, u)=g_{i}\left(t,-y^{*}\left(t-\tau_{i}(t)\right)\right)$ for $t \geq 0$ and $u<-y^{*}\left(t-\tau_{i}(t)\right)$. Define the set $S(t)$ by

$$
S(t)=\left\{\varphi \in P C(t): \varphi(\theta) \geq-y^{*}(t+\theta) \text { for }-\tau(t) \leq \theta<0, \varphi(0)>-y^{*}(t)\right\} .
$$

Naturally, the set $S=\left\{\varphi \in P C: \varphi(\theta) \geq-y^{*}(\theta)\right.$ for $\left.\theta \in[-\bar{\tau}, 0), \varphi(0)>-y^{*}(0)\right\}$ is now taken as the set of admissible initial conditions for the transformed equation. We should note that (1.1) has the form (2.2) where $t \mapsto f_{i}\left(t, x_{t}^{i}\right)$ may have jump discontinuities at points $t$ such that $t-\tau_{i}(t)=t_{k}$ for $k \in \mathbb{N}$.

As usual, the solution $y^{*}(t)$ of (1.2) is said to be GAS in the set of solutions of (1.2) with initial conditions in $P C_{0}^{+}$if and only if the zero solution of (1.1), with $g_{i}$ as in (2.4), is GAS in the set of solutions of (1.1) with initial conditions in $S$.

For the present framework of DDEs with linear impulses (2.2), the assumption set adopted in [31] can be summarized as follows:

(A1) the sequence $P_{n}=\prod_{k=1}^{n}\left(1+b_{k}\right)$ is positive and bounded.

(A2) there exist piecewise continuous functions $\lambda_{1, i}, \lambda_{2, i}:[0, \infty) \rightarrow[0, \infty)$ such that

$$
-\lambda_{1, i}(t) \mathcal{M}_{t}^{i}(\varphi) \leq f_{i}\left(t, \varphi_{\left[-\tau_{i}(t), 0\right]}\right) \leq \lambda_{2, i}(t) \mathcal{M}_{t}^{i}(-\varphi), \quad t \geq 0, \varphi \in P C^{i}(t)
$$

where $\mathcal{M}_{t}^{i}(\varphi)=\max \left\{0, \sup _{\theta \in\left[-\tau_{i}(t), 0\right]} \varphi(\theta)\right\}$, for $i=1, \ldots, m$;

(A3) there exists $T>0$ such that

$$
l\left(\alpha_{1}, \alpha_{1}^{*}\right) l\left(\alpha_{2}, \alpha_{2}^{*}\right)<1,
$$

where $l:\left\{(z, w) \in \mathbb{R}^{2}: z \geq w \geq 0\right\} \rightarrow \mathbb{R}$ is defined by

$$
l(z, w)= \begin{cases}w \min \left\{1, z-\frac{w}{2}\right\}, & w \leq 1 \\ \min \left\{w, z-\frac{1}{2}\right\}, & w>1\end{cases}
$$


and the coefficients $\alpha_{j}:=\alpha_{j}(T)$ and $\alpha_{j}^{*}:=\alpha_{j}^{*}(T)$ are given by

$$
\begin{aligned}
& \alpha_{j}(T)=\sup _{t \geq T} \int_{t-\tau(t)}^{t} \sum_{i=1}^{m} \lambda_{j, i}(s) \mathrm{e}^{\int_{t-\tau(t)}^{s} a(u) d u} B_{i}(s) d s, \quad j=1,2 \\
& \alpha_{j}^{*}(T)=\sup _{t \geq T} \int_{t-\tau(t)}^{t} \sum_{i=1}^{m} \lambda_{j, i}(s) \mathrm{e}^{-\int_{s}^{t} a(u) d u} B_{i}(s) d s, \quad j=1,2,
\end{aligned}
$$

and

$$
B_{i}(t):=\max _{\theta \in\left[-\tau_{i}(t), 0\right]}\left(\prod_{k: t+\theta \leq t_{k}<t}\left(1+b_{k}\right)^{-1}\right), i=1, \ldots, m .
$$

The above condition (A2), known as Yorke-type condition (see [36]), implies that $f_{i}(t, 0)=$ 0 for $t \geq 0$ and $i=1, \ldots, m$, thus $x=0$ is an equilibrium of (2.2). In (2.10), the standard convention that a product $B_{i}(t)$ is equal to one if the number of factors is zero is used.

Applying the stability results in $[31,32]$ to $(2.2)$, we obtain the following result:

Theorem 2.2. [31] Assume (A1)-(A3), where $a(t)$ is a continuous, nonnegative function with $\int_{0}^{\infty} a(t) d t=\infty$ and $f_{i}$ is piecewise continuous and continuous in $\varphi$, for all $i$. Then the zero solution of $(2.2)$ is GAS.

We remind the reader that it is useful to have criteria to easily check whether (2.6) is satisfied or not. The following result is given in [31, Theorem 3.2].

Theorem 2.3. [31] For $l(z, w)$ as in (2.7) and constants $\alpha_{j}, \alpha_{j}^{*}>0$ such that $\alpha_{j} \geq \alpha_{j}^{*}$, $j=1,2$, the estimate (2.6) is satisfied if one of the following conditions holds:

(i) $\alpha_{1}^{*} \alpha_{2}^{*}<1$; (ii) $\alpha_{1} \alpha_{2}<(3 / 2)^{2}$.

From Theorem 2.2, a corollary for the case of DDEs without impulses is stated as follows:

Corollary 2.1. Let $a(t)$ be a continuous, nonnegative function with $\int_{0}^{\infty} a(t) d t=\infty$ and $f_{i}(t, \varphi)$, for $1 \leq i \leq m$. Assume (A2)-(A3), with $\alpha_{j}, \alpha_{j}^{*}, j=1,2$, given by (2.8), (2.9) where $B_{i}(t) \equiv 1$ for all $i$. Then the zero solution of

$$
x^{\prime}(t)+a(t) x(t)=\sum_{i=1}^{m} f_{i}\left(t, x_{t}^{i}\right), \quad t \geq 0
$$

is $G A S$.

\section{Global stability for periodic models}

In this section, we give some improvements of Theorem 2.2 for the case of periodic impulsive DDEs (2.2), with possible extensions to other situations.

For periodic impulsive models, more precisely under the hypotheses (H1)-(H3), the restriction of $\left(P_{n}\right)_{n \in \mathbb{N}}$ bounded in (A1) is equivalent to the condition $\prod_{k=1}^{p}\left(1+b_{k}\right) \leq 1$, which is not convenient from a view point of applications. Our first goal is to replace (A1) by the less restrictive constraint (H4). For that purpose, we observe that hypothesis (A1) was used in [31, Theorem 3.1] only to prove that all non-oscillatory solutions $x(t)$ of $(2.2)$ converge to zero as $t \rightarrow \infty$. We recall that a solution $x(t)$ is oscillatory if it is not eventually zero and has arbitrarily large zeros; otherwise, $x(t)$ is called non-oscillatory. In this way, to show that Theorem 2.2 still holds with (A1) replaced by (H4), it suffices to prove the following lemma: 
Lemma 3.1. Assume (H2)-(H4) and that, for each $i=1, \ldots, m, f_{i}(t, \varphi)$ is piecewise continuous and continuous in $\varphi$, and satisfies

$$
\left(A 2^{*}\right) \text { for } t \geq 0 \text { and } \varphi \in P C^{i}(t), f_{i}(t, \varphi) \leq 0 \text { if } \varphi \geq 0 \text { and } f_{i}(t, \varphi) \geq 0 \text { if } \varphi \leq 0 \text {. }
$$

If $a(t)$ is a continuous nonnegative $\omega$-periodic function not identically zero, then all nonoscillatory solutions of (2.2) converge to zero as $t \rightarrow \infty$.

Proof. Let $x(t)$ be a non-oscillatory solution of (2.2) and assume that $x(t)>0$ for $t \gg 0$ (the situation is analogous if $x(t)<0$ for $t \gg 0$ ). Consider the nonnegative jump function

$$
\Theta(t):=\prod_{k: 0 \leq t_{k}<t}\left(1+b_{k}\right), \quad t \geq 0
$$

From [35, Lemma 3.1], the function

$$
y(t)=\Theta(t)^{-1} x(t), \quad t \geq 0,
$$

is continuous and satisfies

$$
y^{\prime}(t)+a(t) y(t)=\Theta(t)^{-1} \sum_{i=1}^{m} f_{i}\left(t, x_{t}^{i}\right), \quad t \geq 0, t \neq t_{k} .
$$

Consequently, $y(t)>0$ for large $t$ and, from (3.3) and $\left(\mathrm{A} 2^{*}\right), y^{\prime}(t)+a(t) y(t) \leq 0$ for $t \gg 0, t \neq t_{k}$. Define

$$
A(t)=\int_{0}^{t} a(u) d u
$$

Clearly, $A(\infty):=\int_{0}^{\infty} a(u) d u=\infty$. As $t \mapsto y(t) \mathrm{e}^{A(t)}$ is continuous, positive, and $\left(y(t) \mathrm{e}^{A(t)}\right)^{\prime} \leq$ 0 for $t \gg 0, t \neq t_{k}$, there is $w \geq 0$ such that $e^{A(t)} y(t) \searrow w$ as $t \rightarrow \infty$.

On the other hand, denoting by $[t / \omega]$ the integer part of $t / \omega>0$, from (H2), (H3), (3.2) and the conditions imposed on $a(t)$, for large $t$ we have

$$
\begin{aligned}
\mathrm{e}^{A(t)} y(t) & =\mathrm{e}^{A(t)} \Theta(t)^{-1} x(t) \\
& =\left(\mathrm{e}^{A(\omega)} \prod_{k=1}^{p}\left(1+b_{k}\right)^{-1}\right)^{[t / \omega]} \exp \left(\int_{[t / \omega] \omega}^{t} a(u) d u\right)\left(\prod_{k:[t / \omega] \omega<t_{k}<t}\left(1+b_{k}\right)^{-1}\right) x(t) \\
& \geq\left(\mathrm{e}^{A(\omega)} \prod_{k=1}^{p}\left(1+b_{k}\right)^{-1}\right)^{[t / \omega]}\left(\min _{1 \leq j \leq p} \prod_{k=1}^{j}\left(1+b_{k}\right)^{-1}\right) x(t)
\end{aligned}
$$

From (H4), we have

$$
\lim _{t \rightarrow \infty}\left(\mathrm{e}^{A(\omega)} \prod_{k=1}^{p}\left(1+b_{k}\right)^{-1}\right)^{[t / \omega]}=\infty
$$

and (H3) implies that

$$
\min _{1 \leq j \leq p} \prod_{k=1}^{j}\left(1+b_{k}\right)^{-1}>0 .
$$

Finally, as $\lim _{t \rightarrow \infty} y(t) \mathrm{e}^{A(t)}=w \in \mathbb{R}$, we conclude that $\lim _{t \rightarrow \infty} x(t)=0$. 
Now, from [32, Theorem 2.2], [31, Theorem 3.1] and the above previous lemma, we obtain the announced improvement of Theorem 2.2 as follows:

Theorem 3.1. Assume the hypotheses (H2)-(H4), (A2), (A3), with $a(t) \not \equiv 0$ a continuous nonnegative $\omega$-periodic function and $f_{i}$ piecewise continuous and continuous in $\varphi$, for all $i$. Then the zero solution of (2.2) is GAS.

The above proof of Lemma 3.1 can be applied to equations (2.2) with $a(t)$ not necessarily periodic, as far as $\lim _{t \rightarrow \infty} \mathrm{e}^{A(t)} \Theta(t)^{-1}=\infty$. This observation and again [32, Theorem 2.2] lead to the following statement:

Theorem 3.2. Consider (2.2) where: $0 \leq t_{1}<t_{2}<\cdots<t_{k} \rightarrow \infty, b_{k}>-1, k \in \mathbb{N} ; a(t)$ is continuous and nonnegative; $f_{i}$ are piecewise continuous and continuous in $\varphi$, for all $i$. Assume the hypotheses (A2), (A3) and

$$
\lim _{t \rightarrow \infty} \mathrm{e}^{A(t)} \Theta(t)^{-1}=\infty,
$$

where $\Theta(t), A(t)$ are defined by (3.1), (3.4). Then the zero solution of (2.2) is GAS.

In the case of DDEs with linear impulses, a simple change of variables allows us to transfer the impulses to the "growth functions" $f_{i}$, and therefore to reduce $(2.2)$ to a DDE without impulses as in (2.11). Next, in view of our purposes, we restrict the scope of our analysis to models with time-dependent discrete delays of the form (1.1). In particular, we shall see that hypothesis (H4) can in fact be eliminated.

Theorem 3.3. Consider (1.1), where $a:[0, \infty) \rightarrow[0, \infty)$ is a continuous $\omega$-periodic function with $a(t) \not \equiv 0, \beta_{i}, \tau_{i}:[0, \infty) \rightarrow[0, \infty)$ are continuous with $\tau_{i}(t)$ bounded, $g_{i}:[0, \infty) \times \mathbb{R} \rightarrow \mathbb{R}$ is piecewise continuous and continuous in the second variable, $1 \leq i \leq m$, and $\left(b_{k}\right)_{k \in \mathbb{N}} \subset \mathbb{R}$. Assume (H2),(H3) and

(A2') there exist piecewise continuous functions $\lambda_{1, i}, \lambda_{2, i}:[0, \infty) \rightarrow[0, \infty)$ such that

$$
-\lambda_{1, i}(t) \max \{u, 0\} \leq \beta_{i}(t) g_{i}(t, u) \leq \lambda_{2, i}(t) \max \{-u, 0\}, \quad t \geq 0, u \in \mathbb{R}
$$

(A3') condition (A3) is satisfied with $B_{i}(t)$ in (2.10) replaced by

$$
B_{i}(t):=\prod_{k: t-\tau_{i}(t) \leq t_{k}<t}\left(1+b_{k}\right)^{-1}, \quad i=1, \ldots, m .
$$

Then the zero solution of (1.1) is GAS.

Proof. For the jump function $\Theta(t)$ defined above, the change of variables (3.2) transforms (1.1) into a DDE in $P C$ with no impulses of the form (2.11), with $f_{i}$ given by

$$
f_{i}(t, \psi)=\Theta(t)^{-1} \beta_{i}(t) g_{i}\left(t, \Theta\left(t-\tau_{i}(t)\right) \psi\left(-\tau_{i}(t)\right)\right)
$$

for $t \geq 0, \psi \in P C^{i}(t), 1 \leq i \leq m$. Since $\Theta(t)>0$, clearly $\mathcal{M}_{t}^{i}\left(\Theta\left(t-\tau_{i}(t)\right) \psi\right)=\Theta(t-$ $\left.\tau_{i}(t)\right) \mathcal{M}_{t}^{i}(\psi)$. Now, for $t \geq 0$ and $\psi \in P C^{i}(t)$, we have $\psi\left(-\tau_{i}(t)\right) \leq \mathcal{M}_{t}^{i}(\psi)$ and $-\psi\left(-\tau_{i}(t)\right) \leq$ $\mathcal{M}_{t}^{i}(-\psi)$. By the assumption (3.6), each $f_{i}$ satisfies (A2) with $\lambda_{j, i}(t)$ replaced by $\mu_{j, i}(t)=$ $\Theta(t)^{-1} \Theta\left(t-\tau_{i}(t)\right) \lambda_{j, i}(t)=B_{i}(t) \lambda_{j, i}(t)$, where $B_{i}(t)$ is as $(3.7)$, for $1 \leq i \leq m, j=1,2$. Now, Corollary 2.1 gives the result. 
The above Theorem 3.2 concerns equations which are not periodic. Observe also that for models with no impulses the constraint (3.5) reduces to $\int_{0}^{\infty} a(t) d t=\infty$. For (1.1) with $a(t)$ not necessarily periodic, it is clear that Theorem 3.3 holds true as far as $\int_{0}^{\infty} a(t) d t=\infty$.

Theorem 3.4. Consider (1.1), where $a, \beta_{i}, \tau_{i}:[0, \infty) \rightarrow[0, \infty)$ are continuous with $\tau_{i}(t)$ bounded, $g_{i}:[0, \infty) \times \mathbb{R} \rightarrow \mathbb{R}$ is piecewise continuous and continuous in the second variable, $1 \leq i \leq m$, and $b_{k} \in(-1, \infty), k \in \mathbb{N}$. Assume (A2'), (A3') and $\int_{0}^{\infty} a(t) d t=\infty$. Then the zero solution of (1.1) is GAS.

Remark 3.1. As an important illustration of more general models, keep in mind that Theorem 3.4 can be applied to almost periodic impulsive models of hematopoiesis.

\section{Stability of the impulsive hematopoiesis model}

In this section, we study the stability of an $\omega$-periodic solution $y^{*}(t)$ of (1.2). We start with some uniform lower and upper bounds for $y^{*}(t)$.

\subsection{Uniform estimates for periodic solutions}

Assume (H1)-(H4), and let $y^{*}(t)$ be a positive $\omega$-periodic solution of (1.2), whose existence is given in Theorem 2.1. Denote

$$
\underline{y}^{*}:=\inf _{t \in[0, \omega]}\left\{y^{*}(t)\right\} \quad \text { and } \quad \bar{y}^{*}:=\sup _{t \in[0, \omega]}\left\{y^{*}(t)\right\} .
$$

Usually, it is not possible to explicitly compute periodic solutions, thus it is important to obtain estimates for uniform bounds of $y^{*}(t)$ which do not depend on its a priori knowledge. For this purpose, in the next results we present some upper and lower uniform bounds for $y^{*}(t)$, based either on the integral averages of the functions $a(t)$ and $\beta(t):=\sum_{i=1}^{m} \beta_{i}(t)$, or on the ratio $\beta(t) / a(t)$. In fact, the first result below recovers the estimates for the periodic solution established in [18] in the case of the non-impulsive model.

Proposition 4.1. Assume (H1)-(H4). Then any positive $\omega$-periodic solution $y^{*}(t)$ of (1.2) satisfies

$$
\mathfrak{m}_{1}:=\frac{\mathrm{e}^{-A(\omega)} M \underline{B} \beta}{1+(M \beta \bar{B})^{n}} \leq y^{*}(t) \leq M \beta \bar{B}=: \mathfrak{M}_{1}, \quad \forall t \geq 0
$$

where

$$
\begin{gathered}
\underline{B}=\min \left\{1, \prod_{k=j}^{j+l-1}\left(1+b_{k}\right)^{-1}: j=1, \ldots, p, l=0, \ldots, p\right\} \\
\bar{B}=\max \left\{1, \prod_{k=j}^{j+l-1}\left(1+b_{k}\right)^{-1}: j=1, \ldots, p, l=0, \ldots, p\right\} \\
M=\left(\prod_{k=1}^{p}\left(1+b_{k}\right)^{-1}-\mathrm{e}^{-A(\omega)}\right)^{-1}, \quad \text { and } \\
\beta=\int_{0}^{\omega} \sum_{i=1}^{m} \beta_{i}(s) d s, \quad A(\omega)=\int_{0}^{\omega} a(u) d u .
\end{gathered}
$$


Proof. Let $y^{*}(t)$ be a positive $\omega$-periodic solution of (1.2) and consider $z^{*}(t):=\Theta(t)^{-1} y^{*}(t)$, where as before we write $\Theta(t)=\prod_{k: 0 \leq t_{k}<t}\left(1+b_{k}\right)$. From [35, Lemma 3.1], we know that the function $z^{*}(t)$ is continuous and satisfies

$$
z^{* \prime}(t)+a(t) z^{*}(t)=\Theta(t)^{-1} \sum_{i=1}^{m} \frac{\beta_{i}(t)}{1+y^{*}\left(t-\tau_{i}(t)\right)^{n}}, \quad t \geq 0, t \neq t_{k} .
$$

Fix $t \neq t_{k}$. For $A(t)$ as in (3.4), multiplying by $\mathrm{e}^{A(t)}$ and integrating over $[t, t+\omega]$, we obtain

$$
\int_{t}^{t+\omega}\left[z^{*}(s) \mathrm{e}^{A(s)}\right]^{\prime} d s=\int_{t}^{t+\omega} \Theta(s)^{-1} \sum_{i=1}^{m} \frac{\beta_{i}(s) \mathrm{e}^{A(s)}}{1+y^{*}\left(s-\tau_{i}(s)\right)^{n}} d s
$$

which is equivalent to

$$
\begin{aligned}
\Theta(t+\omega)^{-1} y^{*}(t+\omega) \mathrm{e}^{A(t+\omega)}-\Theta(t)^{-1} y^{*}(t) \mathrm{e}^{A(t)} & \\
& =\int_{t}^{t+\omega} \Theta(s)^{-1} \sum_{i=1}^{m} \frac{\beta_{i}(s) \mathrm{e}^{A(s)}}{1+y^{*}\left(s-\tau_{i}(s)\right)^{n}} d s
\end{aligned}
$$

As $y^{*}(t)$ and $a(t)$ are $\omega$-periodic functions and (H2) holds, we obtain

$$
\begin{aligned}
y^{*}(t) \mathrm{e}^{A(t)} \Theta(t)^{-1}[ & \left.\left(\prod_{k=1}^{p}\left(1+b_{k}\right)^{-1}\right) \mathrm{e}^{A(\omega)}-1\right] \\
& =\int_{t}^{t+\omega} \Theta(s)^{-1} \sum_{i=1}^{m} \frac{\beta_{i}(s) \mathrm{e}^{A(s)}}{1+y^{*}\left(s-\tau_{i}(s)\right)^{n}} d s
\end{aligned}
$$

and consequently

$y^{*}(t)=\left(\mathrm{e}^{A(\omega)} \prod_{k=1}^{p}\left(1+b_{k}\right)^{-1}-1\right)^{-1} \int_{t}^{t+\omega}\left(\prod_{k: t \leq t_{k}<s}\left(1+b_{k}\right)^{-1}\right) \sum_{i=1}^{m} \frac{\beta_{i}(s) \mathrm{e}^{\int_{t}^{s} a(u) d u}}{1+y^{*}\left(s-\tau_{i}(s)\right)^{n}} d s(4$

On the one hand, we deduce

$$
\begin{aligned}
y^{*}(t) & \leq\left(\mathrm{e}^{A(\omega)} \prod_{k=1}^{p}\left(1+b_{k}\right)^{-1}-1\right)^{-1} \bar{B} \mathrm{e}^{A(\omega)} \int_{t}^{t+\omega} \sum_{i=1}^{m} \frac{\beta_{i}(s)}{1+y^{*}\left(s-\tau_{i}(s)\right)^{n}} d s \\
& \leq\left(\prod_{k=1}^{p}\left(1+b_{k}\right)^{-1}-\mathrm{e}^{-A(\omega)}\right)^{-1} \bar{B} \int_{t}^{t+\omega} \sum_{i=1}^{m} \beta_{i}(s) d s=M \bar{B} \beta .
\end{aligned}
$$

On the other hand, from (4.4) and the previous estimate, we have

$$
\begin{aligned}
y^{*}(t) & \geq\left(\mathrm{e}^{A(\omega)} \prod_{k=1}^{p}\left(1+b_{k}\right)^{-1}-1\right)^{-1} \int_{t}^{t+\omega}\left(\prod_{k: t \leq t_{k}<s}\left(1+b_{k}\right)^{-1}\right) \sum_{i=1}^{m} \frac{\beta_{i}(s) \mathrm{e}^{\int_{t}^{s} a(u) d u}}{1+(M \bar{B} \beta)^{n}} d s \\
& \geq \frac{\left(\prod_{k=1}^{p}\left(1+b_{k}\right)^{-1}-\mathrm{e}^{-A(\omega)}\right)^{-1} \mathrm{e}^{-A(\omega)} \underline{B}}{1+(M \bar{B} \beta)^{n}} \int_{t}^{t+\omega} \sum_{i=1}^{m} \beta_{i}(s) d s=\frac{M \mathrm{e}^{-A(\omega)} \underline{B} \beta}{1+(M \bar{B} \beta)^{n}} .
\end{aligned}
$$


Proposition 4.2. Assume (H1)-(H4) with $a(t)>0, \beta(t):=\sum_{i=1}^{m} \beta_{i}(t) \not \equiv 0$, and define $c_{1} \geq 0, c_{2}>0$ by

$$
c_{1}=\min _{t \in[0, \omega]} \frac{\beta(t)}{a(t)}, \quad c_{2}=\max _{t \in[0, \omega]} \frac{\beta(t)}{a(t)} .
$$

A positive $\omega$-periodic solution $y^{*}(t)$ of (1.2) satisfies the estimates

$$
\mathfrak{m}_{2} \leq y^{*}(t) \leq \mathfrak{M}_{2}, \quad t \geq 0,
$$

where

$$
\mathfrak{M}_{2}=c_{2} \mathrm{e}^{-A(\omega)} M \bar{B}\left(\mathrm{e}^{A(\omega)}-1\right), \quad \mathfrak{m}_{2}=c_{1} \mathrm{e}^{-A(\omega)} M \underline{B} \frac{\mathrm{e}^{A(\omega)}-1}{1+\mathfrak{M}_{2}{ }^{n}},
$$

where $\underline{B}, \bar{B}, A(\omega)$ and Mare as in Proposition 4.1.

In particular, for the equation without impulses

$$
y^{\prime}(t)+a(t) y(t)=\sum_{i=1}^{m} \frac{\beta_{i}(t)}{1+y\left(t-\tau_{i}(t)\right)^{n}}, \quad t \geq 0,
$$

with a $(t), \beta_{i}(t), \tau_{i}(t)$ as above, it holds

$$
\frac{c_{1}}{1+c_{2}^{n}} \leq y^{*}(t) \leq c_{2}, \quad t \geq 0 .
$$

Proof. One proceeds as in the above proof, noting that in (4.4) one can use the estimate

$$
\int_{t}^{t+\omega} \sum_{i=1}^{m} \frac{\beta_{i}(s) \mathrm{e}_{t}^{s} a(u) d u}{1+y^{*}\left(s-\tau_{i}(s)\right)^{n}} d s \leq c_{2} \int_{t}^{t+\omega} a(s) \mathrm{e}^{\int_{t}^{s} a(u) d u} d s=c_{2}\left(\mathrm{e}^{A(\omega)}-1\right) .
$$

A similar argument is applied to obtain the uniform lower bound $\mathfrak{m}_{2}$, so computations are omitted.

Combining the above two propositions, new estimates are obtained as follows:

Proposition 4.3. Assume (H1)-(H4) with $a(t)>0$ and $\beta(t):=\sum_{i=1}^{m} \beta_{i}(t) \not \equiv 0$. With the notations in Propositions 4.1 and 4.2, set

$$
\mathfrak{M}=\min \left\{\mathfrak{M}_{1}, \mathfrak{M}_{2}\right\}, \quad \mathfrak{m}=\frac{\mathrm{e}^{-A(\omega)} M \underline{B}}{1+\mathfrak{M}^{n}} \max \left\{\beta, c_{1}\left(\mathrm{e}^{A(\omega)}-1\right)\right\} .
$$

Then, a positive $\omega$-periodic solution $y^{*}(t)$ of (1.2) satisfies the estimates

$$
\mathfrak{m} \leq y^{*}(t) \leq \mathfrak{M}, \quad t \geq 0 .
$$

To proceed with the study of the asymptotic behaviour of solutions to (1.2), as explained in Section 2, we consider (1.1) with $g_{i}$ defined by (2.4). The next step is to find functions $\lambda_{1, i}(t), \lambda_{2, i}(t)$ such that the Yorke condition (3.6) holds for each function $g_{i}, i=1, \ldots, m$. In this way, in order to choose adequate functions $\lambda_{j, i}(t)$, it is convenient to consider the situations $n \leq 1$ and $n>1$ separately. 


\subsection{Case $n \in(0,1]$}

Lemma 4.1. Assume (H1)-(H4). If $n \in(0,1]$, then the functions $g_{i}$ defined in (2.4) satisfy the Yorke conditions (3.6), for $i=1, \ldots, m$, with $\lambda_{1, i}, \lambda_{2, i}:[0, \infty) \rightarrow[0, \infty)$ given by

$$
\lambda_{1, i}(t)=\frac{n \beta_{i}(t) y^{*}\left(t-\tau_{i}(t)\right)^{n-1}}{\left[1+y^{*}\left(t-\tau_{i}(t)\right)^{n}\right]^{2}}, \quad \lambda_{2, i}(t)=\frac{\beta_{i}(t) y^{*}\left(t-\tau_{i}(t)\right)^{n-1}}{1+y^{*}\left(t-\tau_{i}(t)\right)^{n}}, \quad t \geq 0 .
$$

Proof. For each $i=1, \ldots, m$ and $g_{i}:[0, \infty) \times \mathbb{R} \rightarrow \mathbb{R}$ as in (2.4), we have

$$
\frac{\partial g_{i}}{\partial u}(t, u)=-\frac{n\left(u+y^{*}\left(t-\tau_{i}(t)\right)\right)^{n-1}}{\left[1+\left(u+y^{*}\left(t-\tau_{i}(t)\right)\right)^{n}\right]^{2}}<0, \quad \forall t \geq 0, \quad \forall u>-y^{*}\left(t-\tau_{i}(t)\right),
$$

and

$$
\frac{\partial^{2} g_{i}}{\partial u^{2}}(t, u)=\frac{n\left(u+y^{*}\left(t-\tau_{i}(t)\right)\right)^{n-2}\left[1-n+(1+n)\left(u+y^{*}\left(t-\tau_{i}(t)\right)\right)^{n}\right]}{\left[1+\left(u+y^{*}\left(t-\tau_{i}(t)\right)\right)^{n}\right]^{3}} .
$$

Consequently, for each $t \geq 0$ and $i=1, \ldots, m$, the function $u \mapsto g_{i}(t, u)$ is decreasing on $\left[-y^{*}\left(t-\tau_{i}(t)\right), \infty\right)$ and, as $n \in(0,1], \frac{\partial^{2} g_{i}}{\partial u^{2}}(t, u)>0$ for all $u>-y^{*}\left(t-\tau_{i}(t)\right)$. Now it is clear that

$$
0 \geq g_{i}(t, u) \geq \frac{\partial g_{i}}{\partial u}(t, 0) u=-\frac{n y^{*}\left(t-\tau_{i}(t)\right)^{n-1}}{\left[1+y^{*}\left(t-\tau_{i}(t)\right)^{n}\right]^{2}} u, \quad \forall t \geq 0, \quad \forall u \geq 0,
$$

and

$0 \leq g_{i}(t, u) \leq \frac{g_{i}\left(t,-y^{*}\left(t-\tau_{i}(t)\right)\right)}{-y^{*}\left(t-\tau_{i}(t)\right)} u=-\frac{y^{*}\left(t-\tau_{i}(t)\right)^{n-1}}{1+y^{*}\left(t-\tau_{i}(t)\right)^{n}} u, \quad \forall t \geq 0, \forall u \in\left[-y^{*}\left(t-\tau_{i}(t)\right), 0\right]$.

Hence, (3.6) is satisfied with $\lambda_{j, i}(t)(j=1,2$ and $1 \leq i \leq m)$ as in the statement of the lemma.

This lemma shows that $g_{i}(t, u)$ satisfies (3.6) for $t \geq 0, u \in\left[-y^{*}\left(t-\tau_{i}(t)\right), \infty\right)$, hence (A2') holds. Therefore, by Theorem 3.3 we obtain the following stability criterion:

Theorem 4.1. Assume (H1)-(H4) and $n \in(0,1]$ in (1.2). In addition, suppose that there is $T>0$ such that $l\left(\alpha_{1}, \alpha_{1}^{*}\right) l\left(\alpha_{2}, \alpha_{2}^{*}\right)<1$, for $l(z, w)$ defined by (2.7) and the coefficients $\alpha_{j}$ and $\alpha_{j}^{*}$ given by $\alpha_{j}:=\alpha_{j T}=\sup _{t \geq T} \alpha_{j}(t)$ and $\alpha_{j}^{*}:=\alpha_{j T}^{*}=\sup _{t \geq T} \alpha_{j}^{*}(t)$ and $\alpha_{j}(t)=\alpha_{j}^{*}(t) \mathrm{e}^{\int_{t-\tau(t)}^{t} a(u) d u}$, $j=1,2$, where

$$
\begin{aligned}
& \alpha_{1}^{*}(t)=\int_{t-\tau(t)}^{t} \sum_{i=1}^{m} \frac{n \beta_{i}(s) y^{*}\left(s-\tau_{i}(s)\right)^{n-1}}{\left[1+y^{*}\left(s-\tau_{i}(s)\right)^{n}\right]^{2}} B_{i}(s) \mathrm{e}^{-\int_{s}^{t} a(u) d u} d s \\
& \alpha_{2}^{*}(t)=\int_{t-\tau(t)}^{t} \sum_{i=1}^{m} \frac{\beta_{i}(s) y^{*}\left(s-\tau_{i}(s)\right)^{n-1}}{1+y^{*}\left(s-\tau_{i}(s)\right)^{n}} B_{i}(s) \mathrm{e}^{-\int_{s}^{t} s a(u) d u} d s
\end{aligned}
$$

and $B_{i}(t)$ is given by (3.7). Then the positive $\omega$-periodic solution $y^{*}(t)$ of (1.2) is GAS (in $\left.P C_{0}^{+}\right)$, i.e., $y^{*}(t)$ is stable and any positive solution $y(t)$ of $(1.2)$ satisfies $\lim _{t \rightarrow \infty}\left(y(t)-y^{*}(t)\right)=$ 0 . 
Remark 4.1. As the $\omega$-periodic solution $y^{*}(t)$ is usually unknown, it is desirable to have some estimates not depending on $y^{*}(t)$, in order to apply the stability result in Theorem 4.1.

Since $s \mapsto \frac{n s^{n-1}}{\left(1+s^{n}\right)^{2}}$ and $s \mapsto \frac{s^{n-1}}{1+s^{n}}$ are decreasing on $(0, \infty)$ and $\mathfrak{m} \leq \underline{y}^{*}$ by Proposition 4.3 , then $\lambda_{1, i}(t), \lambda_{2, i}(t)$ defined in Lemma 4.1 satisfy the inequalities

$$
\lambda_{1, i}(t) \leq \frac{n \beta_{i}(t) \underline{y}^{* n-1}}{\left[1+\underline{y}^{* n}\right]^{2}} \leq \frac{n \beta_{i}(t) \mathfrak{m}^{n-1}}{\left[1+\mathfrak{m}^{n}\right]^{2}}, \quad \lambda_{2, i}(t) \leq \frac{\beta_{i}(t) \underline{y}^{* n-1}}{1+\underline{y}^{* n}} \leq \frac{\beta_{i}(t) \mathfrak{m}^{n-1}}{1+\mathfrak{m}^{n}},
$$

$t \geq 0, i=1, \ldots, m$. Hence, one can replace $\alpha_{1}^{*}(t), \alpha_{2}^{*}(t)$ in Theorem 4.1 by

$$
\alpha_{1}^{*}(t)=\frac{n \mathfrak{m}^{n-1}}{\left[1+\mathfrak{m}^{n}\right]^{2}} \mathcal{B}(t), \quad \alpha_{2}^{*}(t)=\frac{\mathfrak{m}^{n-1}}{1+\mathfrak{m}^{n}} \mathcal{B}(t)
$$

respectively, where

$$
\mathcal{B}(t):=\int_{t-\tau(t)}^{t} \sum_{i=1}^{m} \beta_{i}(s) B_{i}(s) \mathrm{e}^{-\int_{s}^{t} a(u) d u} d s,
$$

and obtain the same conclusions. Now, note that the $\omega$-periodicity of the functions $a(t), \beta_{i}(t)$, $B_{i}(t)$ and $\tau(t)$ implies that $\mathcal{B}(t)$ is $\omega$-periodic, hence $\sup _{t \geq T} \mathcal{B}(t)=\sup _{t \in[0, \omega]} \mathcal{B}(t)$.

Corollary 4.1. Under (H1)-(H4) and for $n \in(0,1]$ in $(1.2)$, if

$$
\frac{\mathfrak{m}^{n-1} \sqrt{n}}{\left[1+\mathfrak{m}^{n}\right]^{3 / 2}} \mathcal{B}<\max \left\{1, \frac{3}{2} \mathrm{e}^{-\mathcal{A}}\right\}
$$

where

$$
\begin{aligned}
& \mathcal{A}:=\sup _{t \in[0, \omega]} \int_{t-\tau(t)}^{t} a(u) d u \\
& \mathcal{B}:=\sup _{t \in[0, \omega]} \int_{t-\tau(t)}^{t} \sum_{i=1}^{m} \beta_{i}(s) B_{i}(s) \mathrm{e}^{-\int_{s}^{t} a(u) d u} d s,
\end{aligned}
$$

then the positive $\omega$-periodic solution $y^{*}(t)$ is $G A S$ (in $P C_{0}^{+}$). Moreover, the above statements also hold with $\mathfrak{m}$ replaced by $\underline{y}^{*}$ or any other uniform lower bound for $y^{*}(t)$.

Proof. With $\alpha_{j}^{*}(t)$ given by (4.12), as before take $\alpha_{j}(t)=\alpha_{j}^{*}(t) \mathrm{e}^{\int_{t-\tau(t)}^{t} a(u) d u}$. With the notations $\alpha_{j}=\sup _{t \geq T} \alpha_{j}(t), \alpha_{j}^{*}=\sup _{t \geq T} \alpha_{j}^{*}(t)(j=1,2)$, we have $\alpha_{j} \leq \alpha_{j}^{*} \mathrm{e}^{\mathcal{A}}(j=1,2)$ and it is clear that $\sqrt{\alpha_{1}^{*} \alpha_{2}^{*}} \leq \frac{\sqrt{n} \mathfrak{m}^{n-1}}{\left[1+\mathfrak{m}^{n}\right]^{3 / 2}} \mathcal{B}$. Thus (4.14) implies that either $\alpha_{1}^{*} \alpha_{2}^{*}<1$ or $\alpha_{1} \alpha_{2}<(3 / 2)^{2}$. The result follows now from Theorem 2.3.

Remark 4.2. In the above corollary, observe that the effect of the impulses is shown in the definition of $\mathcal{B}$, whereas the effect of the delays appears in both $\mathcal{A}, \mathcal{B}$.

Remark 4.3. If $a(t)$ is a positive function and $c:=\max _{t \in[0, \omega]}\left(\sum_{i=1}^{m} \beta_{i}(t) B_{i}(t)\right) / a(t)$, one deduces that

$$
\mathcal{B} \leq c\left(1-\mathrm{e}^{-\mathcal{A}}\right)
$$

for $\mathcal{A}, \mathcal{B}$ as in (4.15). Also, for $c_{2}=\max _{t \in[0, \omega]} \frac{\beta(t)}{a(t)}$ as in (4.5) and $\bar{B}_{0}:=\max _{1 \leq i \leq m} \max _{t \in[0, \omega]} B_{i}(t)$, one obtains $\mathcal{B} \leq c_{2} \bar{B}_{0}\left(1-\mathrm{e}^{-\mathcal{A}}\right)$. 


\subsection{Case $n>1$}

We now consider the impulsive delayed hematopoiesis model (1.2) with $n>1$.

Lemma 4.2. Assume (H1)-(H4).

If $n>1$, then hypothesis (A2') holds for $g_{i}$ defined by (2.4) and $\lambda_{1, i}, \lambda_{2, i}:[0, \infty) \rightarrow$ $[0, \infty), i=1, \ldots, m$, given by one of the following situations:

(i) $\left\{\begin{array}{l}\lambda_{1, i}(t)=\frac{n \beta_{i}(t) y^{*}\left(t-\tau_{i}(t)\right)^{n-1}}{\left[1+y^{*}\left(t-\tau_{i}(t)\right)^{n}\right]^{2}} \\ \lambda_{2, i}(t)=\beta_{i}(t) \rho_{n}\end{array}\right.$, for the case $\underline{y}^{*} \geq\left(\frac{n-1}{n+1}\right)^{\frac{1}{n}}$;

(ii) $\left\{\begin{array}{l}\lambda_{1, i}(t)=\beta_{i}(t) \rho_{n} \\ \lambda_{2, i}(t)=\frac{n \beta_{i}(t) y^{*}\left(t-\tau_{i}(t)\right)^{n-1}}{\left[1+y^{*}\left(t-\tau_{i}(t)\right)^{n}\right]^{2}}\end{array}\right.$, for the case $\bar{y}^{*} \leq\left(\frac{n-1}{n+1}\right)^{\frac{1}{n}}$.

(iii) $\lambda_{1, i}(t)=\lambda_{2, i}(t)=\beta_{i}(t) \rho_{n}$, for all cases,

where $\rho_{n}:=\frac{(n+1)^{2}}{4 n}\left(\frac{n-1}{n+1}\right)^{\frac{n-1}{n}}$ and $\underline{y}^{*}, \bar{y}^{*}$ are defined by (4.1).

Proof. For each $i=1, \ldots, m$, consider $g_{i}(t, u)$ defined on $[0, \infty) \times \mathbb{R}$ as in (2.4). We have $\frac{\partial g_{i}}{\partial u}(t, u)<0$ for all $t \geq 0$ and $u>-y^{*}\left(t-\tau_{i}(t)\right)$, and consequently, for each $t \geq 0$, the function $u \mapsto g_{i}(t, u)$ is decreasing on $\left[-y^{*}\left(t-\tau_{i}(t)\right), \infty\right)$. Since $n>1$, from (4.9) and (4.10) we conclude that $u_{0}(t):=-y^{*}\left(t-\tau_{i}(t)\right)+\left(\frac{n-1}{n+1}\right)^{\frac{1}{n}}$ is the unique inflection point of $u \mapsto g_{i}(t, u)$ on $\left(-y^{*}\left(t-\tau_{i}(t)\right), \infty\right)$ with $\frac{\partial^{2} g_{i}}{\partial u^{2}}(t, u)>0$ for $u>u_{0}(t)$, and $\frac{\partial^{2} g_{i}}{\partial u^{2}}(t, u)<0$ for $u \in\left(-y^{*}\left(t-\tau_{i}(t)\right), u_{0}(t)\right)$ (see Figure 1 ).

Next, consider the case (i), that is, assume that $\left(\frac{n-1}{n+1}\right)^{\frac{1}{n}} \leq \underline{y}^{*}$. In this situation, the inflection point $u_{0}(t)$ is nonpositive, for all $t \geq 0$, thus

$$
0 \geq g_{i}(t, u) \geq \frac{\partial g_{i}}{\partial u}(t, 0) u=-\frac{n y^{*}\left(t-\tau_{i}(t)\right)^{n-1}}{\left[1+y^{*}\left(t-\tau_{i}(t)\right)^{n}\right]^{2}} u, \quad \forall u \geq 0,
$$

and

$$
0 \leq g_{i}(t, u) \leq \frac{\partial g_{i}}{\partial u}\left(t, u_{0}(t)\right) u=-\rho_{n} u, \forall u \in\left[-y^{*}\left(t-\tau_{i}(t)\right), 0\right] .
$$

Hence, each $g_{i}$ satisfies the Yorke condition (3.6) with $\lambda_{1, i}(t), \lambda_{2, i}(t)$ defined by (i).

Analogously, in the case $\bar{y}^{*} \leq\left(\frac{n-1}{n+1}\right)^{\frac{1}{n}}$, we can prove that each $g_{i}$ satisfies the Yorke condition (A2') with $\lambda_{1, i}(t), \lambda_{2, i}(t)$ defined by (ii).

We further observe that in any situation

$$
\frac{\partial^{2} g_{i}}{\partial u^{2}}(t, u)\left(u-u_{0}(t)\right)>0, \quad \forall u \in\left(-y^{*}\left(t-\tau_{i}(t)\right), \infty\right) \backslash\left\{u_{0}(t)\right\}
$$


Consequently

$$
0 \geq g_{i}(t, u) \geq \frac{\partial g_{i}}{\partial u}\left(t, u_{0}(t)\right) u=-\rho_{n} u, \quad \forall u>0
$$

and

$$
0 \leq g_{i}(t, u) \leq \frac{\partial g_{i}}{\partial u}\left(t, u_{0}(t)\right) u=-\rho_{n} u, \quad \forall u \in\left[-y^{*}\left(t-\tau_{i}(t)\right), 0\right),
$$

thus each $g_{i}$ satisfies (3.6) with $\lambda_{1, i}(t), \lambda_{2, i}(t)$ defined by (iii).

The figure below illustrates the situation in Lemma 4.2(i).

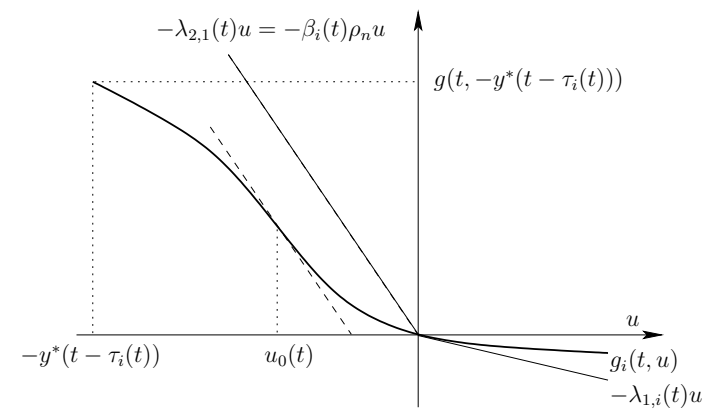

Figure 1: Graph of the functions $u \mapsto g_{i}(t, u), u \mapsto-\lambda_{j i}(t) u$, and $u \mapsto-\beta_{i}(t) \rho_{n} u$ for situation (i) with $t>0$ fixed; $u_{0}(t)$ is the inflexion point of $u \mapsto g_{i}(t, u)$.

We now use the previous lemma and apply Theorem 3.3 to (1.2) with $n>1$.

Theorem 4.2. Consider (1.2) with $n>1$ and assume (H1)-(H4). For $T>0$, define the coefficients $\gamma=\gamma_{T}=\sup _{t \geq T} \gamma(t), \gamma^{*}=\gamma_{T}^{*}=\sup _{t \geq T} \gamma(t) \mathrm{e}^{-\int_{t-\tau(t)}^{t} a(u) d u}$ and $\alpha=\alpha_{T}=\sup _{t \geq T} \alpha(t)$, $\alpha^{*}=\alpha_{T}^{*}=\sup _{t \geq T} \alpha(t) \mathrm{e}^{-\int_{t-\tau(t)}^{t} a(u) d u}$, where

$$
\gamma(t)=\rho_{n} \int_{t-\tau(t)}^{t} \sum_{i=1}^{m} \beta_{i}(s) B_{i}(s) \mathrm{e}^{\int_{t-\tau(t)}^{s} a(u) d u} d s
$$

and

$$
\alpha(t)=\int_{t-\tau(t)}^{t} \sum_{i=1}^{m} \frac{n \beta_{i}(s) y^{*}\left(s-\tau_{i}(s)\right)^{n-1}}{\left[1+y^{*}\left(s-\tau_{i}(s)\right)^{n}\right]^{2}} B_{i}(s) \mathrm{e}^{\int_{t-\tau(t)}^{s} a(u) d u} d s
$$

for $\rho_{n}=\frac{(n+1)^{2}}{4 n}\left(\frac{n-1}{n+1}\right)^{\frac{n-1}{n}}$ and $B_{i}(t)$ as in (3.7). Let $l(z, w)$ be as in (2.7). Then, there exists a unique positive $\omega$-periodic solution $y^{*}(t)$ of $(1.2)$, which is GAS if one of the following conditions holds:

(i) $l\left(\alpha, \alpha^{*}\right) l\left(\gamma, \gamma^{*}\right)<1$ and $\underline{y}^{*} \geq\left(\frac{n-1}{n+1}\right)^{\frac{1}{n}} ;$

(ii) $l\left(\alpha, \alpha^{*}\right) l\left(\gamma, \gamma^{*}\right)<1$ and $\bar{y}^{*} \leq\left(\frac{n-1}{n+1}\right)^{\frac{1}{n}}$;

(iii) $l\left(\gamma, \gamma^{*}\right)<1$ 
Remark 4.4. In case $\underline{y}^{*}$ and $\bar{y}^{*}$ are unknown, but either $\mathfrak{m} \geq\left(\frac{n-1}{n+1}\right)^{\frac{1}{n}}$ or $\mathfrak{M} \leq\left(\frac{n-1}{n+1}\right)^{\frac{1}{n}}$, for $\mathfrak{m}, \mathfrak{M}$ defined as in (4.8), we can proceed as follows. For $n>1$, the function $s \mapsto \frac{s^{n-1}}{\left(1+s^{n}\right)^{2}}$ is increasing on $\left[0,\left(\frac{n-1}{n+1}\right)^{\frac{1}{n}}\right]$ and decreasing on $\left[\left(\frac{n-1}{n+1}\right)^{\frac{1}{n}}, \infty\right)$. Thus, if $\mathfrak{m} \geq\left(\frac{n-1}{n+1}\right)^{\frac{1}{n}}$, then $\underline{y}^{*} \geq \mathfrak{m}$ from Proposition 4.3, and therefore

$$
\lambda_{1, i}(t) \leq \frac{n \beta_{i}(t) \underline{y}^{* n-1}}{\left[1+\underline{y}^{* n}\right]^{2}} \leq \frac{n \beta_{i}(t) \mathfrak{m}^{n-1}}{\left[1+\mathfrak{m}^{n}\right]^{2}}, \quad t \geq 0, i=1, \ldots, m,
$$

for $\lambda_{1, i}(t)$ defined in Lemma 4.2 (i). Analogously, if $\mathfrak{M} \leq\left(\frac{n-1}{n+1}\right)^{\frac{1}{n}}$, then $\bar{y}^{*} \leq \mathfrak{M}$ and

$$
\lambda_{2, i}(t) \leq \frac{n \beta_{i}(t) \bar{y}^{* n-1}}{\left[1+\bar{y}^{* n}\right]^{2}} \leq \frac{n \beta_{i}(t) \mathfrak{M}^{n-1}}{\left[1+\mathfrak{M}^{n}\right]^{2}}, \quad t \geq 0, i=1, \ldots, m,
$$

for $\lambda_{2, i}(t)$ defined in Lemma 4.2 (ii). Consequently, reasoning as for the proof of Corollary 4.1, the following results are derived. The details are omitted.

Corollary 4.2. Assume (H1)-(H4) with $n>1$ in (1.2), let $y^{*}(t)$ be the positive $\omega$-periodic solution of (1.2) and define $\mathcal{A}, \mathcal{B}$ as in (4.15). Then $y^{*}(t)$ is $G A S$ (in $P C_{0}^{+}$) if one of the following conditions holds:

(i) $\frac{\sqrt{n \rho_{n} \mathfrak{m}^{n-1}}}{1+\mathfrak{m}^{n}} \mathcal{B}<\max \left\{1, \frac{3}{2} \mathrm{e}^{-\mathcal{A}}\right\}$ and $\mathfrak{m} \geq\left(\frac{n-1}{n+1}\right)^{\frac{1}{n}}$;

(ii) $\frac{\sqrt{n \rho_{n} \mathfrak{M}^{n-1}}}{1+\mathfrak{M}^{n}} \mathcal{B}<\max \left\{1, \frac{3}{2} \mathrm{e}^{-\mathcal{A}}\right\}$ and $\mathfrak{M} \leq\left(\frac{n-1}{n+1}\right)^{\frac{1}{n}}$;

(iii) $\rho_{n} \mathcal{B}<\max \left\{1, \frac{3}{2} \mathrm{e}^{-\mathcal{A}}\right\}$.

Naturally, these stability criteria also hold if, instead of $\mathfrak{m}$ and $\mathfrak{M}$, other lower and upper uniform estimates for $y^{*}(t)$, respectively, are used. Remark 4.2 applies to this case as well.

\subsection{Case of delays multiple of the period}

Now, we pursue our analysis with the situation of (1.2) with time independent delays multiple of the period, $\tau_{i}(t) \equiv q_{i} \omega$, where $q_{i}$ are positive integers, $i=1, \ldots, m$ :

$$
\left\{\begin{array}{l}
y^{\prime}(t)+a(t) y(t)=\sum_{i=1}^{m} \frac{\beta_{i}(t)}{1+y\left(t-q_{i} \omega\right)^{n}}, 0 \leq t \neq t_{k}, \\
y\left(t_{k}^{+}\right)-y\left(t_{k}\right)=b_{k} y\left(t_{k}\right), \quad k \in \mathbb{N} .
\end{array}\right.
$$

See [24] for the case $m=1$ and $n \in \mathbb{N}$. As before, the cases $0<n \leq 1$ and $n>1$ are treated separately.

For $\bar{q}:=\max _{1 \leq i \leq m} q_{i}$, we have $\tau(t)=\bar{q} \omega, \mathcal{A}=\bar{q} A(\omega)$ and the constants $\alpha_{j}, \alpha_{j}^{*}$, defined in Theorem $4.1, \bar{j}=1,2$, satisfy

$$
\alpha_{j}=\alpha_{j}^{*} \mathrm{e}^{\bar{q} \int_{0}^{\omega} a(u) d u}=\alpha_{j}^{*} \mathrm{e}^{\bar{q} A(\omega)} .
$$

In what follows we establish upper estimates for the coefficients $\alpha_{1}^{*}, \alpha_{2}^{*}$ in Theorem 4.1. 
Theorem 4.3. Assume (H1)-(H4) with $n \in(0,1]$ and $\tau_{i}(t)=q_{i} \omega\left(q_{i} \in \mathbb{N}\right)$. Then there is a positive $\omega$-periodic solution $y^{*}(t)$ of $(4.22)$, which is $G A S$ (in $\mathrm{PC}_{0}^{+}$), if

$$
\frac{\underline{y}^{* n-1} \sqrt{n}}{\sqrt{1+\underline{y}^{* n}}} \mathcal{E}<\max \left\{1, \frac{3}{2} \mathrm{e}^{-\bar{q} A(\omega)}\right\}
$$

where

$$
\mathcal{E}:=B \bar{y}^{*}\left(1-\mathrm{e}^{-\bar{q} A(\omega)}\right)\left[1-\left(1-\mathrm{e}^{-A(\omega)}\right)^{-1} \sum_{k=1}^{p} \min \left\{b_{k}, 0\right\}\right],
$$

$B_{0}=\prod_{k=1}^{p}\left(1+b_{k}\right)^{-1}, B=\max \left\{B_{0}^{\bar{q}}, B_{\overline{0}}^{\underline{q}}\right\}, \bar{q}=\max _{1 \leq i \leq m} q_{i}, \underline{q}=\min _{1 \leq i \leq m} q_{i}, A(\omega)=\int_{0}^{\omega} a(u) d u$, and $\underline{y}^{*}, \bar{y}^{*}$ are defined by (4.1).

In particular, if there are no impulses or the impulses $b_{1}, b_{2}, \ldots, b_{p}$ are all nonnegative, then $y^{*}(t)$ is GAS if

$$
\frac{\underline{y}^{* n-1} \sqrt{n}}{\sqrt{1+\underline{y}^{* n}}} \bar{y}^{*}\left(1-\mathrm{e}^{-\bar{q} A(\omega)}\right)<\max \left\{1, \frac{3}{2} \mathrm{e}^{-\bar{q} A(\omega)}\right\} .
$$

Proof. From (H2) and (H3), for $t \geq 0,1 \leq i \leq m$ and $B_{i}(t)$ as in (3.7), we have

$$
B_{i}(t)=\prod_{k: t-q_{i} \omega \leq t_{k}<t}\left(1+b_{k}\right)^{-1} \equiv B_{0}^{q_{i}} \leq B
$$

Since $y^{*}(t)$ is an $\omega$-periodic solution of (1.2), then

$$
\left(y^{*}\right)^{\prime}(t)+a(t) y^{*}(t)=\sum_{i=1}^{m} \frac{\beta_{i}(t)}{1+y^{*}(t)^{n}}=\frac{\beta(t)}{1+y^{*}(t)^{n}}, \quad \text { for } 0 \leq t \neq t_{k},
$$

for $\beta(t)=\sum_{i=1}^{m} \beta_{i}(t)$. Recalling that $y^{*}(t)$ has possible jumps at the points $t_{k}$, for $\alpha_{1}^{*}=\alpha_{1 T}^{*}$ defined in Theorem 4.1, from (4.26) we derive

$$
\begin{aligned}
\alpha_{1}^{*} & =n \sup _{t \geq T} \int_{t-\bar{q} \omega}^{t} \sum_{i=1}^{m} \frac{\beta_{i}(s) y^{*}(s)^{n-1}}{\left[1+y^{*}(s)^{n}\right]^{2}} B_{i}(s) \mathrm{e}^{-\int_{s}^{t} a(u) d u} d s \\
& \leq \frac{n B \underline{y}^{* n-1}}{1+\underline{y}^{* n}} \sup _{t \geq T} \int_{t-\bar{q} \omega}^{t} \frac{d}{d s}\left[y^{*}(s) \mathrm{e}^{\int_{t}^{s} a(u) d u}\right] d s \\
& =\frac{n B \underline{y}^{* n-1}}{1+\underline{y}^{* n}} \sup _{t \geq T}\left[y^{*}(t)\left(1-\mathrm{e}^{-\bar{q} A(\omega)}\right)-\sum_{k: t_{k} \in[t-\bar{q} \omega, t)} b_{k} y^{*}\left(t_{k}\right) \mathrm{e}^{-\int_{t_{k}}^{t} a(u) d u}\right] .
\end{aligned}
$$

Let $b_{k}^{-}=\max \left\{-b_{k}, 0\right\}=-\min \left\{b_{k}, 0\right\}$. We have

$$
\begin{aligned}
& \sum_{k: t_{k} \in[t-\bar{q} \omega, t)} b_{k}^{-} y^{*}\left(t_{k}\right) \mathrm{e}^{-\int_{t_{k}}^{t} a(u) d u} \\
= & \left(\sum_{k: t_{k} \in[t-\omega, t)} b_{k}^{-} y^{*}\left(t_{k}\right) \mathrm{e}^{-\int_{t_{k}}^{t} a(u) d u}\right)\left(1+\mathrm{e}^{-A(\omega)}+\cdots+\mathrm{e}^{-(\bar{q}-1) A(\omega)}\right) \\
\leq & \bar{y}^{*}\left(\sum_{k=1}^{p} b_{k}^{-}\right) \frac{1-\mathrm{e}^{-\bar{q} A(\omega)}}{1-\mathrm{e}^{-A(\omega)}} .
\end{aligned}
$$


The estimates (4.27) and (4.28) yield

$$
\alpha_{1}^{*} \leq \frac{n B \underline{y}^{* n-1} \bar{y}^{*}}{1+\underline{y}^{* n}}\left(1-\mathrm{e}^{-\bar{q} A(\omega)}\right)\left[1-\left(1-\mathrm{e}^{-A(\omega)}\right)^{-1} \sum_{k=1}^{p} \min \left\{b_{k}, 0\right\}\right]=\frac{n \underline{y}^{* n-1}}{1+\underline{y}^{* n}} \mathcal{E}=: \sigma_{1}^{*} .
$$

In a similar way, for $\alpha_{2}^{*}=\alpha_{2 T}^{*}$ defined in Theorem 4.1 we also obtain

$$
\alpha_{2}^{*} \leq B \underline{y}^{* n-1} \bar{y}^{*}\left(1-\mathrm{e}^{-\bar{q} A(\omega)}\right)\left[1-\left(1-\mathrm{e}^{-A(\omega)}\right)^{-1} \sum_{k=1}^{p} \min \left\{b_{k}, 0\right\}\right]=\underline{y}^{* n-1} \mathcal{E}=: \sigma_{2}^{*} .
$$

Clearly, condition (4.24) is equivalent to

$$
\sigma_{1}^{*} \sigma_{2}^{*}<\max \left\{1, \frac{9}{4} \mathrm{e}^{-2 \bar{q} A(\omega)}\right\}
$$

As $\alpha_{j}=\alpha_{j}^{*} \mathrm{e}^{\bar{q} A(\omega)}\left(\right.$ see (4.23)) for $\alpha_{j}$ defined in Theorem 4.1, $j=1,2$, condition (4.29) implies that

$$
\alpha_{1}^{*} \alpha_{2}^{*}<1 \quad \text { or } \quad \alpha_{1} \alpha_{2}<\frac{9}{4} .
$$

Now, the conclusion follows from Theorem 4.1 because, by Theorem 2.3, (4.30) implies $l\left(\alpha_{1}, \alpha_{1}^{*}\right) l\left(\alpha_{2}, \alpha_{2}^{*}\right)<1$.

Observe also that with $b_{k} \geq 0(1 \leq k \leq p)$, then $B \leq 1$ and $b_{k}^{-}=0(1 \leq k \leq p)$.

As already mentioned, we emphasize that other stability criteria are deduced if in (4.24) and (4.25), we replace $y^{*}$ and $\bar{y}^{*}$ by $\mathfrak{m}$ and $\mathfrak{M}$ or any other lower and upper estimates for $y^{*}(t)$, respectively (see Subsection 4.1 ).

Remark 4.5. Consider (4.22) with no impulses, $a(t)$ positive, $c_{2}$ as in (4.5) and $0<n \leq 1$. With the above notations, one has $\mathcal{E}=\bar{y}^{*}\left(1-\mathrm{e}^{-\bar{q} A(\omega)}\right), c_{1}\left(1-\mathrm{e}^{-\bar{q} A(\omega)}\right) \leq \mathcal{B} \leq c_{2}\left(1-\mathrm{e}^{-\bar{q} A(\omega)}\right)$. For the constants

$$
C_{1}:=\frac{\underline{y}^{* n-1} \sqrt{n}}{\left[1+\underline{y}^{* n}\right]^{3 / 2}} \mathcal{B}, \quad C_{2}:=\frac{\underline{y}^{* n-1} \sqrt{n}}{\sqrt{1+\underline{y}^{* n}}} \mathcal{E},
$$

one deduces the estimates

$$
C_{1} \leq \frac{c_{2}}{\bar{y}^{*}\left[1+\underline{y}^{* n}\right]} C_{2} \quad \text { and } \quad C_{2} \leq \frac{\bar{y}^{*}\left[1+\underline{y}^{* n}\right]}{c_{1}} C_{1} \leq \frac{c_{2}}{c_{1}}\left[1+\underline{y}^{* n}\right] C_{1} .
$$

Therefore, the constraints $C_{1}<\max \left\{1, \frac{3}{2} \mathrm{e}^{-\bar{q} A(\omega)}\right\}$, imposed by (4.14) in Corollary 4.1, and $C_{2}<\max \left\{1, \frac{3}{2} \mathrm{e}^{-\bar{q} A(\omega)}\right\}$, imposed by (4.24) in Theorem 4.3, are not always comparable so one should check which one is more convenient to use. Of course, for the autonomous case without impulses, in which $y^{*}(t) \equiv y^{*}$ is a positive equilibrium, $C_{1}=C_{2}$ and the two criteria coincide.

Similarly to the case $0<n \leq 1$ studied above, consider now (4.22) with $n>1$. The coefficients $\gamma, \gamma^{*}$ and $\alpha, \alpha^{*}$ in Theorem 4.2 satisfy $\gamma=\gamma^{*} \mathrm{e}^{\bar{q} A(\omega)}$ and $\alpha=\alpha^{*} \mathrm{e}^{\bar{q} A(\omega)}$, with $\alpha^{*}$ now given by

$$
\begin{aligned}
& \gamma^{*}=\rho_{n} \sup _{t \geq T} \int_{t-\bar{q} \omega}^{t} \sum_{i=1}^{m} \beta_{i}(s) B_{i}(s) \mathrm{e}^{-\int_{s}^{t} a(u) d u} d s \\
& \alpha^{*}=\sup _{t \geq T} \int_{t-\bar{q} \omega}^{t} \sum_{i=1}^{m} \frac{n \beta_{i}(s) y^{*}(s)^{n-1}}{\left[1+y^{*}(s)^{n}\right]^{2}} B_{i}(s) \mathrm{e}^{-\int_{s}^{t} a(u) d u} d s
\end{aligned}
$$


Arguing as in the proof of Theorem 4.3, below we establish alternative upper estimates for $\gamma^{*}, \alpha^{*}$ which are easier to check.

Theorem 4.4. Assume (H1)-(H4) with $n>1$ and $\tau_{i}(t)=q_{i} \omega\left(q_{i} \in \mathbb{N}\right)$. Then the positive $\omega$-periodic solution $y^{*}(t)$ of $(4.22)$ is $G A S$ (in $P C_{0}^{+}$) if one of the following conditions holds:

(i) $\sqrt{\rho_{n}(n-1)^{\frac{n-1}{n}}\left(1+\bar{y}^{* n}\right)} \mathcal{E}<\max \left\{1, \frac{3}{2} \mathrm{e}^{-\bar{q} A(\omega)}\right\}$ and $\underline{y}^{*} \geq\left(\frac{n-1}{n+1}\right)^{\frac{1}{n}}$;

(ii) $\sqrt{n \rho_{n} \bar{y}^{* n-1}} \mathcal{E}<\max \left\{1, \frac{3}{2} \mathrm{e}^{-\bar{q} A(\omega)}\right\}$ and $\bar{y}^{*} \leq\left(\frac{n-1}{n+1}\right)^{\frac{1}{n}}$;

(iii) $\rho_{n}\left(1+\bar{y}^{* n}\right) \mathcal{E}<\max \left\{1, \frac{3}{2} \mathrm{e}^{-\bar{q} A(\omega)}\right\}$,

where $\rho_{n}=\frac{(n+1)^{2}}{4 n}\left(\frac{n-1}{n+1}\right)^{\frac{n-1}{n}}, A(\omega)=\int_{0}^{\omega} a(u) d u, \mathcal{E}$ is defined by (4.25), and $\underline{y}^{*}, \bar{y}^{*}$ are defined by (4.1). In particular, if there are no impulses or the impulses $b_{1}, b_{2}, \ldots, b_{p}$ are all nonnegative, then $y^{*}(t)$ is GAS if either (i), (ii) or (iii) holds with $\mathcal{E}=\bar{y}^{*}\left(1-\mathrm{e}^{-\bar{q} A(\omega)}\right)$. Moreover, in (i), (ii) and (iii) one can replace $\underline{y}^{*}$ and $\bar{y}^{*}$ by any other uniform lower and upper bounds for $y^{*}(t)$, respectively.

Proof. Again, take $B_{0}=\prod_{k=1}^{p}\left(1+b_{k}\right)^{-1}, B=\max \left\{B_{0}^{\bar{q}}, B_{0}^{\underline{q}}\right\}$. Since $y^{*}(t)$ is an $\omega$-periodic solution of (1.2), for $\gamma^{*}$ defined as in (4.31),

$$
\begin{aligned}
\gamma^{*} & \leq \rho_{n} B\left(1+\bar{y}^{* n}\right) \sup _{t \geq T} \int_{t-\bar{q} \omega}^{t} \sum_{i=1}^{m} \frac{\beta_{i}(s)}{1+y^{*}(s)^{n}} \mathrm{e}^{-\int_{s}^{t} a(u) d u} d s \\
& =\rho_{n} B\left(1+\bar{y}^{* n}\right) \sup _{t \geq T} \int_{t-\bar{q} \omega}^{t} \frac{d}{d s}\left[y^{*}(s) \mathrm{e}^{\int_{t}^{s} a(u) d u}\right] d s
\end{aligned}
$$

and, arguing as in the proof of Theorem 4.3, we conclude that

$$
\gamma^{*} \leq \rho_{n}\left(1+\bar{y}^{* n}\right) \mathcal{E}=: \sigma_{\gamma}^{*} .
$$

For $\alpha^{*}$ given in (4.31), we also have

$$
\alpha^{*} \leq n B \sup _{t \geq T} \int_{t-\bar{q} \omega}^{t} \frac{y^{*}(s)^{n-1}}{1+y^{*}(s)^{n}} \sum_{i=1}^{m} \frac{\beta_{i}(s)}{1+y^{*}(s)^{n}} \mathrm{e}^{-\int_{s}^{t} a(u) d u} d s
$$

and, since $s \mapsto \frac{s^{n-1}}{1+s^{n}}$ is increasing on $\left[0,(n-1)^{\frac{1}{n}}\right]$ and decreasing on $\left[(n-1)^{\frac{1}{n}}, \infty\right)$, we conclude that

$$
\alpha^{*} \leq B(n-1)^{\frac{n-1}{n}} \sup _{t \geq T} \int_{t-\bar{q} \omega}^{t} \frac{d}{d s}\left[y^{*}(s) \mathrm{e}^{\int_{t}^{s} a(u) d u}\right] d s \quad \text { if } \quad \underline{y}^{*} \geq\left(\frac{n-1}{n+1}\right)^{\frac{1}{n}}
$$

and

$$
\alpha^{*} \leq n B \frac{\bar{y}^{* n-1}}{1+\bar{y}^{* n}} \sup _{t \geq T} \int_{t-\bar{q} \omega}^{t} \frac{d}{d s}\left[y^{*}(s) \mathrm{e}^{\int_{t}^{s} a(u) d u}\right] d s \quad \text { if } \quad \bar{y}^{*} \leq\left(\frac{n-1}{n+1}\right)^{\frac{1}{n}} .
$$

At this step, we can perform computations as in the proof of Theorem 4.3, so details are omitted. In this way, we obtain

$$
\alpha^{*} \leq(n-1)^{\frac{n-1}{n}} \mathcal{E}=: \sigma_{1}^{*} \quad \text { if } \quad \underline{y}^{*} \geq\left(\frac{n-1}{n+1}\right)^{\frac{1}{n}}
$$


and

$$
\alpha^{*} \leq \frac{n \bar{y}^{* n-1}}{1+\bar{y}^{* n}} \mathcal{E}=: \sigma_{2}^{*} \quad \text { if } \quad \bar{y}^{*} \leq\left(\frac{n-1}{n+1}\right)^{\frac{1}{n}} .
$$

Finally, in case $\underline{y}^{*} \geq\left(\frac{n-1}{n+1}\right)^{\frac{1}{n}}$, condition (i) is equivalent to

$$
\sigma_{\gamma}^{*} \sigma_{1}^{*}<\max \left\{1, \frac{9}{4} \mathrm{e}^{-2 \bar{q} A(\omega)}\right\}
$$

which implies that

$$
\alpha^{*} \gamma^{*}<1 \quad \text { or } \quad \alpha \gamma<\frac{9}{4}
$$

for $\alpha=\alpha^{*} \mathrm{e}^{\bar{q} A(\omega)}$ and $\gamma=\gamma^{*} \mathrm{e}^{\bar{q} A(\omega)}$. Consequently $l\left(\alpha, \alpha^{*}\right) l\left(\gamma, \gamma^{*}\right)<1$ and the conclusion follows from Theorem 4.2.

Analogously, in case $\bar{y}^{*} \leq\left(\frac{n-1}{n+1}\right)^{\frac{1}{n}}$, condition (ii) is equivalent to

$$
\sigma_{\gamma}^{*} \sigma_{2}^{*}<\max \left\{1, \frac{9}{4} \mathrm{e}^{-2 \bar{q} A(\omega)}\right\}
$$

which again implies that $l\left(\alpha, \alpha^{*}\right) l\left(\gamma, \gamma^{*}\right)<1$. With similar arguments, if (iii) holds, then $\gamma^{*}<1$ or $\gamma<3 / 2$, and the global asymptotic stability of $y^{*}(t)$ folows from Theorem 4.2.

Remark 4.6. Whenever the results in Subsections 4.2-4.4 require the a priori knowledge of a uniform lower or upper bound for $y^{*}(t)$, of course one can apply previously Propositions 4.1, 4.2 or 4.3 to obtain such uniform bounds. Nevertheless, in Subsections 4.3 and 4.4, the above criteria involving only $\gamma$ or $\gamma^{*}$ in conditions (iii) are much easier to apply. In Section 5 , we shall however consider an example where (iii) is not satisfied, still the global asymptotic stability of $y^{*}(t)$ can be derived by application of either (i) or (ii). The remark below also emphasizes the importance of these alternative criteria.

Remark 4.7. Note that $\lim _{n \rightarrow \infty} \rho_{n}=\infty, \lim _{n \rightarrow 1^{+}} \rho_{n}=1$, and that $\mathfrak{M}$ defined by (4.8) and $\mathcal{B}$ defined by (4.15) are independent of $n$. Below, we stress two situations where Corollary 4.2(iii) is not applicable, however either the criterion in (i) or in (ii) can be invoked. These ideas will be exploited in more detail with a numerical example, see (5.13) in Section 5.

1. If $\mathfrak{M} \in(0,1)$, it is clear that condition (iii) in Corollary 4.2 fails for $n>1$ sufficiently large, whereas condition (ii) holds, because

$$
\lim _{n \rightarrow \infty}\left(\frac{n-1}{n+1}\right)^{\frac{1}{n}}=1 \quad \text { and } \quad \lim _{n \rightarrow \infty} \frac{\sqrt{n \rho_{n} \mathfrak{M}^{n-1}}}{1+\mathfrak{M}^{n}}=0 .
$$

2. With $c_{1}$ as in (4.5) and $\mathfrak{m}$ as in (4.8), if

$$
1+\frac{\mathrm{e}^{-A(\omega)} M \underline{B}}{1+\mathfrak{M}} \max \left\{\beta, c_{1}\left(\mathrm{e}^{A(\omega)}-1\right)\right\}>\frac{\mathcal{B}}{\max \left\{1, \frac{3}{2} \mathrm{e}^{-\mathcal{A}}\right\}}>1,
$$

then, for $n>1$ close enough to 1 , condition (iii) fails and condition (i) holds, in Corollary 4.2. In fact, on the one hand, $\mathcal{B}>\max \left\{1, \frac{3}{2} \mathrm{e}^{-\mathcal{A}}\right\}$ and $\lim _{n \rightarrow 1^{+}} \rho_{n}=1$ imply that condition (iii) fails for $n>1$ close to 1 . On the other hand, since

$$
\lim _{n \rightarrow 1^{+}} \frac{\sqrt{n \rho_{n} \mathfrak{m}^{n-1}}}{1+\mathfrak{m}^{n}} \mathcal{B}=\frac{\mathcal{B}}{1+\frac{\mathrm{e}^{-A(\omega)} M \underline{B} \max \left\{\beta, c_{1}\left(\mathrm{e}^{A(\omega)}-1\right)\right\}}{1+\mathfrak{M}}}<\max \left\{1, \frac{3}{2} \mathrm{e}^{-\mathcal{A}}\right\}
$$


$\lim _{n \rightarrow 1^{+}}\left(\frac{n-1}{n+1}\right)^{\frac{1}{n}}=0$ and $\lim _{n \rightarrow 1^{+}} \mathfrak{m}>0$, then condition (i) holds for $n>1$ close enough to 1.

\section{Particular models}

With the choice of some particular models below, we discuss our results within the context of recent literature. We shall see that our approach leads to sharper criteria than the few ones found in previous works, even in the case of models without impulses.

\subsection{The case $n=1$}

Consider (1.2) with $n=1$, assume (H1)-(H4) with $a(t)$ positive and define $\beta(t):=\sum_{i=1}^{m} \beta_{i}(t)$. Let $y^{*}(t)$ be a positive $\omega$-periodic solution, whose existence is asserted in Theorem 2.1. Consider also the previous terminology for $A(\omega), \bar{B}, \mathcal{A}, \mathcal{B}, \mathcal{E}$, etc.

From Corollary 4.1, if

$$
\frac{1}{\left[1+\underline{y}^{*}\right]^{3 / 2}} \mathcal{B}<\max \left\{1, \frac{3}{2} \mathrm{e}^{-\mathcal{A}}\right\}
$$

then $y^{*}(t)$ is GAS; in particular, this holds true if $\mathcal{B} \leq \max \left\{1, \frac{3}{2} \mathrm{e}^{-\mathcal{A}}\right\}$. From Remark 4.2, we also conclude that

$$
\max _{t \in[0, \omega]}\left(\frac{\beta(t)}{a(t)}\right) \bar{B}\left(1-\mathrm{e}^{-\mathcal{A}}\right) \leq \max \left\{1, \frac{3}{2} \mathrm{e}^{-\mathcal{A}}\right\}
$$

is a sufficient condition for the global attractivity of the positive $\omega$-periodic solution $y^{*}(t)$.

For the special case

$$
\left\{\begin{array}{l}
y^{\prime}(t)+a(t) y(t)=\sum_{i=1}^{m} \frac{\beta_{i}(t)}{1+y\left(t-q_{i} \omega\right)}, 0 \leq t \neq t_{k}, \\
y\left(t_{k}^{+}\right)-y\left(t_{k}\right)=b_{k} y\left(t_{k}\right), \quad k \in \mathbb{N},
\end{array} .\right.
$$

with $q_{i} \in \mathbb{N}$, Theorem 4.3 implies that $y^{*}(t)$ is GAS if

$$
\frac{1}{\left[1+\underline{y}^{*}\right]^{1 / 2}} \mathcal{E}<\max \left\{1, \frac{3}{2} \mathrm{e}^{-\bar{q} A(\omega)}\right\}
$$

and this condition is satisfied if $\mathcal{E} \leq \max \left\{1, \frac{3}{2} \mathrm{e}^{-\bar{q} A(\omega)}\right\}$. For the nonimplusive DDE

$$
y^{\prime}(t)+a(t) y(t)=\sum_{i=1}^{m} \frac{\beta_{i}(t)}{1+y\left(t-q_{i} \omega\right)}, \quad t \geq 0,
$$

with $q_{i} \in \mathbb{N}, \omega>0$ and $a:[0, \infty) \rightarrow(0, \infty), \beta_{i}:[0, \infty) \rightarrow[0, \infty) \omega$-periodic continuous with $\beta(t):=\sum_{i=1}^{m} \beta_{i}(t)$, Proposition 4.2 implies that $\bar{y}^{*} \leq \max _{t \in[0, \omega]}\left(\frac{\beta(t)}{a(t)}\right)$. Either using again Theorem 4.3, or simply from (5.1), it follows that

$$
\max _{t \in[0, \omega]}\left(\frac{\beta(t)}{a(t)}\right)\left(1-\mathrm{e}^{-\bar{q} A(\omega)}\right) \leq \max \left\{1, \frac{3}{2} \mathrm{e}^{-\bar{q} A(\omega)}\right\}
$$

or equivalently

$$
\max _{t \in[0, \omega]}\left(\frac{\beta(t)}{a(t)}\right) \leq \max \left\{\frac{\mathrm{e}^{\bar{q} A(\omega)}}{\mathrm{e}^{\bar{q} A(\omega)}-1}, \frac{\frac{3}{2}}{\mathrm{e}^{\bar{q} A(\omega)}-1}\right\}
$$

is a sufficient condition for the global asymptotic stability of $y^{*}(t)$. 


\subsection{Models with one delay multiple of the period}

Consider a periodic hematopoiesis model with one discrete delay multiple of the period:

$$
\left\{\begin{array}{l}
y^{\prime}(t)+a(t) y(t)=\frac{\beta(t)}{1+y(t-q \omega)^{n}}, 0 \leq t \neq t_{k}, \\
y\left(t_{k}^{+}\right)-y\left(t_{k}\right)=b_{k} y\left(t_{k}\right), \quad k \in \mathbb{N},
\end{array} .\right.
$$

where $n \in(1, \infty), q \in \mathbb{N},\left(t_{k}\right)_{k \in \mathbb{N}}$ is an increasing unbounded real sequence, and $a, \beta$ : $[0, \infty) \rightarrow(0, \infty)$ are $\omega$-periodic continuous functions with $\omega>0$. To the best of our knowledge, few researchs have been devoted to the study of the global asymptotic stability of a periodic solution to a delayed hematopoiesis model with impulses, the work [24] being an exception. Note however that (5.3) was studied in [24] only for the case $n \in \mathbb{N}$.

Applying Theorems 2.1, 4.1 and 4.2(iii) to (5.3), we obtain the following result:

Theorem 5.1. Consider $\omega$-periodic continuous functions $a, \beta:[0, \infty) \rightarrow(0, \infty)$, for some $\omega>0$, and a real sequence $\left(b_{k}\right)$, for which hypotheses (H2)-(H4) are satisfied. Let $B_{0}=$ $\prod_{k=1}^{p}\left(b_{k}+1\right)^{-1}, \rho_{n}=\frac{(n+1)^{2}}{4 n}\left(\frac{n-1}{n+1}\right)^{\frac{n-1}{n}}$ and $\mathfrak{m}$ given by (4.8). Assume that:

(a) either $n>1$ and there exists $T \geq 0$ such that

$$
\rho_{n} B_{0}^{q} \sup _{t \geq T} \int_{t-q \omega}^{t} \beta(s) \mathrm{e}^{-\int_{s}^{t} a(u) d u} d s<\max \left\{1, \frac{3}{2} \mathrm{e}^{-q \int_{0}^{\omega} a(u) d u}\right\}
$$

(b) or $0<n \leq 1$ and there exists $T \geq 0$ such that

$$
\sqrt{n} B_{0}^{q} \sup _{t \geq T} \int_{t-q \omega}^{t} \beta(s) \mathrm{e}^{-\int_{s}^{t} a(u) d u} d s \leq \mathfrak{m} \max \left\{1, \frac{3}{2} \mathrm{e}^{-q \int_{0}^{\omega} a(u) d u}\right\} .
$$

Then (5.3) has a positive $\omega$-periodic solution $y^{*}(t)$ which is GAS.

Proof. For the model (5.3), with $B(t)=B_{1}(t)$ in (3.7), we have $B(t):=\prod_{k: t-q \omega \leq t_{k}<t}\left(b_{k}+\right.$ $1)^{-1} \equiv B_{0}^{q}$. Suppose first that $n>1$. The function $\gamma(t)$ defined by (4.18) assumes the form

$$
\gamma(t)=\int_{t-q \omega}^{t} \rho_{n} \beta(s) B(s) \mathrm{e}^{\int_{t-q \omega}^{s} a(u) d u} d s=\rho_{n} B_{0}^{q} \mathrm{e}^{q \int_{0}^{\omega} a(u) d u} \int_{t-q \omega}^{t} \beta(s) \mathrm{e}^{-\int_{s}^{t} a(u) d u} d s .
$$

Condition (5.4) implies that either $\gamma:=\sup _{t \geq T} \gamma(t)<\frac{3}{2}$ or

$$
\gamma^{*}:=\rho_{n} B_{0}^{q} \sup _{t \geq T} \int_{t-q \omega}^{t} \beta(s) \mathrm{e}^{-\int_{s}^{t} a(u) d u} d s<1 .
$$

From Theorem 2.3, we have $l\left(\gamma, \gamma^{*}\right)<1$ and the conclusion follows from Theorem 4.2.

For $0<n \leq 1$, from (4.12) and (4.13), rough estimates give

$$
\alpha_{1}^{*}(t) \leq n B_{0}^{q} \mathfrak{m}^{-1} \int_{t-q \omega}^{t} \beta(s) \mathrm{e}^{-\int_{s}^{t} a(u) d u} d s, \alpha_{2}^{*}(t) \leq B_{0}^{q} \mathfrak{m}^{-1} \int_{t-q \omega}^{t} \beta(s) \mathrm{e}^{-\int_{s}^{t} a(u) d u} d s,
$$

and with $\alpha_{i}^{*}:=\sup _{t \geq T} \alpha_{i}^{*}(t), i=1,2$, the result follows again from Theorem 2.3. 
Remark 5.1. We observe that in [24] Saker and Alzabut assumed hypotheses (H1), (H3) and the requirement that the impulsive function $t \mapsto \prod_{k: t_{k} \in[0, t)}\left(b_{k}+1\right)$ is $\omega$-periodic. As already mentioned, this latter condition is very restrictive, since it implies (H2) and $\prod_{i=1}^{p}\left(1+b_{k}\right)=$ 1. Under these requirements, in [24, Theorem 4.7] Saker and Alzabut proved the global attractivity of the positive $\omega$-periodic solution $y^{*}(t)$ of $(5.3)$, only for the cases $n=1,2, \ldots$ and with an additional " $3 / 2$-type condition", as follows:

$$
\rho_{n} q \int_{0}^{\omega} \beta(s) d s<\frac{3}{2} \mathrm{e}^{-q \int_{0}^{\omega} a(u) d u} .
$$

Of course, if $B_{0}:=\prod_{i=1}^{p}\left(1+b_{k}\right)=1$, then (5.4) reduces to

$$
\rho_{n} \sup _{t \geq T} \int_{t-q \omega}^{t} \beta(s) \mathrm{e}^{-\int_{s}^{t} a(u) d u} d s<\max \left\{1, \frac{3}{2} \mathrm{e}^{-q \int_{0}^{\omega} a(u) d u}\right\},
$$

which obviously is less restrictive than (5.6). In conclusion, Theorem 5.1 significantly improves the stability criterion established in [24, Theorem 4.7].

\subsection{Models with $n>1$ and no impulses}

To deepen the discussion of our results within the context of the literature, we consider the model (1.2) without impulses, that is,

$$
y^{\prime}(t)+a(t) y(t)=\sum_{i=1}^{m} \frac{\beta_{i}(t)}{1+y\left(t-\tau_{i}(t)\right)^{n}}, t \geq 0,
$$

with $n>1$. As mentioned, the case of (5.7) with $n \leq 1$ has been further analysed in [33]. Recall that, for models without impulses, our setting holds with $B(t)=B_{i}(t) \equiv 1$ for $i=1, \ldots, m$. The result below is an immediate consequence of Corollary 4.2(iii).

Theorem 5.2. Consider (5.7) with $n>1$ and assume (H1). If

$$
\rho_{n} \sup _{t \in[0, \omega]} \int_{t-\tau(t)}^{t} \sum_{i=1}^{m} \beta_{i}(s) \mathrm{e}^{-\int_{s}^{t} a(u) d u} d s<\max \left\{1, \frac{3}{2} \mathrm{e}^{-\mathcal{A}}\right\},
$$

where $\rho_{n}=\frac{(n+1)^{2}}{4 n}\left(\frac{n-1}{n+1}\right)^{\frac{n-1}{n}}$ and $\mathcal{A}:=\sup _{t \in[0, \omega]} \int_{t-\tau(t)}^{t} a(u) d u$, then the positive $\omega$-periodic solution of (5.7) is GAS.

Remark 5.2. For the case $n>1$, in [18, Theorem 3.1] the global asymptotic stability of the positive $\omega$-periodic solution of (5.7) was obtained assuming

$$
(n-1)^{\frac{n-1}{n}} \frac{\mathrm{e}^{A(\omega)}}{\mathrm{e}^{A(\omega)}-1} \int_{0}^{\omega} \sum_{i=1}^{m} \beta_{i}(s) d s \leq 1,
$$

where $A(\omega)=\int_{0}^{\omega} a(u) d u$. However, the present authors have shown in [33] that

$$
\int_{t-\tau(t)}^{t} \sum_{i=1}^{m} \beta_{i}(s) \mathrm{e}^{-\int_{s}^{t} a(u) d u} d s \leq \frac{\mathrm{e}^{A(\omega)}}{\mathrm{e}^{A(\omega)}-1} \int_{0}^{\omega} \sum_{i=1}^{m} \beta_{i}(s) d s .
$$

Moreover, one can easily check that $\rho_{n}<(n-1)^{\frac{n-1}{n}}$ for any $n>1$, thus condition (5.8) is less restrictive than (5.9). This shows that Theorem 5.2 strongly improves the stability criterion presented in [18, Theorem 3.1] for (5.7) with $n>1$. 
Corollary 5.1. Consider (5.7) with $n>1$ and assume (H1) with a(t) a positive function and $\beta(t):=\sum_{i=1}^{m} \beta_{i}(t) \not \equiv 0$.

If either (a)

$$
\rho_{n} \sup _{t \in[0, \omega]} \int_{t-\tau(t)}^{t} \beta(s) d s \leq \max \left\{1, \frac{3}{2} \mathrm{e}^{-\mathcal{A}}\right\}
$$

or (b)

$$
\rho_{n} \max _{t \in[0, \omega]}\left(\frac{\beta(t)}{a(t)}\right)<\frac{1}{1-\mathrm{e}^{-\mathcal{A}}} \max \left\{1, \frac{3}{2} \mathrm{e}^{-\mathcal{A}}\right\}
$$

where $\rho_{n}=\frac{(n+1)^{2}}{4 n}\left(\frac{n-1}{n+1}\right)^{\frac{n-1}{n}}$ and $\mathcal{A}=\sup _{t \in[0, \omega]} \int_{t-\tau(t)}^{t} a(u) d u$, then the positive $\omega$-periodic solution of (5.7) is GAS.

Proof. First, it is clear that (5.10) implies (5.8). Next, observe that

$$
\begin{aligned}
\rho_{n} \sup _{t \in[0, \omega]} \int_{t-\tau(t)}^{t} \beta(s) \mathrm{e}^{-\int_{s}^{t} a(u) d u} d s & =\rho_{n} \sup _{t \in[0, \omega]} \int_{t-\tau(t)}^{t} \frac{\beta(s)}{a(s)} \frac{d}{d s}\left(\mathrm{e}^{\int_{t}^{s} a(u) d u}\right) d s \\
& \leq \rho_{n} \max _{t \in[0, \omega]}\left(\frac{\beta(t)}{a(t)}\right) \sup _{t \in[0, \omega]}\left(1-\mathrm{e}^{-\int_{t-\tau(t)}^{t} a(u) d u}\right) \\
& =\rho_{n} \max _{t \in[0, \omega]}\left(\frac{\beta(t)}{a(t)}\right)\left(1-\mathrm{e}^{-\mathcal{A}}\right)
\end{aligned}
$$

Hence, (5.11) implies (5.8) and the conclusion follows from Theorem 5.2.

Remark 5.3. Consider the nonimpulsive model (5.7) with $m=1$ :

$$
y^{\prime}(t)+a(t) y(t)=\frac{\beta(t)}{1+y(t-\tau(t))^{n}}, \quad t \geq 0,
$$

For (5.12) with $n>1$, Berezansky et. al. [21, Theorem 6.2] established that the positive $\omega$-periodic solution of (5.12) is GAS if one of the following conditions holds:

(a) $\rho_{n} \sup _{t \in[0, \omega]} \int_{t-\tau(t)}^{t} \beta(s) d s<1+\mathrm{e}^{-1} ; \quad$ (b) $\frac{\rho_{n} \bar{\beta}}{\underline{a}}<1$,

where $\underline{a}=\min _{t \in[0, \omega]} a(t)$ and $\bar{\beta}=\max _{t \in[0, \omega]} \beta(t)$. Since $\frac{1}{1-\mathrm{e}^{-\mathcal{A}}} \max \left\{1, \frac{3}{2} \mathrm{e}^{-\mathcal{A}}\right\}>1$, the stability criterion presented in Corollary 5.1(b) strongly improves condition (b) described above. On the other hand, if $\frac{3}{2} \mathrm{e}^{-\mathcal{A}}>1+\mathrm{e}^{-1}$ i.e., if $\mathcal{A} \leq \log \frac{3}{2\left(1+\mathrm{e}^{-1}\right)} \approx 0.0922$, Corollary 5.1(a) improves condition (a) given in [21].

To illustrate the feasibility and efficiency of some of our results, a concrete example is now studied.

Example 5.1. Consider (5.7) with $m=3$ and the delay functions $\tau_{i}(t)=D_{i}+\cos (2 \pi t), i=$ $1,2,3$, with $D_{1}=6, D_{2}=7, D_{3}=15$. Choose $a(t)=1+\frac{1}{2} \cos (2 \pi t), \beta_{1}(t)=\eta_{1}\left(1+\frac{1}{2} \cos (2 \pi t)\right)$, $\beta_{2}(t)=\eta_{2}\left(1+\frac{1}{2} \sin (2 \pi t)\right), \beta_{3}(t)=\eta_{3}\left(1+\frac{1}{2} \cos (2 \pi t)\right)$ with $\eta_{1}, \eta_{2}, \eta_{3}>0$. Eq. (5.7) assumes the form

$$
\begin{aligned}
y^{\prime}(t)= & -\left(1+\frac{1}{2} \cos (2 \pi t)\right) y(t)+\frac{\eta_{1}\left(1+\frac{1}{2} \cos (2 \pi t)\right)}{1+y(t-6-\cos (2 \pi t))^{n}}+\frac{\eta_{2}\left(1+\frac{1}{2} \sin (2 \pi t)\right)}{1+y(t-7-\cos (2 \pi t))^{n}} \\
& +\frac{\eta_{3}\left(1+\frac{1}{2} \cos (2 \pi t)\right)}{1+y(t-15-\cos (2 \pi t))^{n}}, \quad t \geq 0,
\end{aligned}
$$


which is a 1-periodic model.

For (5.13), we take any $n>1$ and define $\beta(t)=\beta_{1}(t)+\beta_{2}(t)+\beta_{3}(t), \beta=\int_{0}^{1} \beta(t) d t=$ $\eta_{1}+\eta_{2}+\eta_{3}$, and the constants $\mathfrak{M}, \mathfrak{m}, c_{1}, c_{2}, \mathcal{A}, \mathcal{B}$ as in the previous sections.

It is easy to see that $\mathfrak{M}=\min \left\{\mathfrak{M}_{1}, \mathfrak{M}_{2}\right\}=\min \left\{\frac{\eta_{1}+\eta_{2}+\eta_{3}}{1-\mathrm{e}^{-1}}, c_{2}\right\}, \eta_{1}+\frac{11}{5} \eta_{2}+\eta_{3} \leq$ $c_{2} \leq \eta_{1}+\frac{23}{10} \eta_{2}+\eta_{3}, \mathfrak{m}=\frac{1}{1+\mathfrak{M}^{n}} \max \left\{\frac{\eta_{1}+\eta_{2}+\eta_{3}}{\mathrm{e}-1}, c_{1}\right\}, \eta_{1}+\frac{2}{5} \eta_{2}+\eta_{3} \leq c_{1} \leq \eta_{1}+\frac{1}{2} \eta_{2}+\eta_{3}$, $A(1)=1, \mathcal{A}=16$, which implies that $1>\frac{3}{2} \mathrm{e}^{-\mathcal{A}}$, and

$$
\underline{\mathcal{B}}:=\left(\eta_{1}+\eta_{3}+\eta_{2} \frac{1}{\mathrm{e}-1}\right)\left(1-\mathrm{e}^{-16}\right) \leq \mathcal{B} \leq\left(\eta_{1}+\eta_{3}+\eta_{2} \frac{1}{1-\mathrm{e}^{-1}}\right)\left(1-\mathrm{e}^{-16}\right)=: \overline{\mathcal{B}} .
$$

All the inequalities given below in this section are easily proven through elementary arguments of calculus. Some however require long computations, which are omitted.

Now, take $\eta_{1}=0.03, \eta_{2}=0.43$ and $\eta_{3}=0.001$. For these values, we claim that the 1-periodic positive solution of (5.13) is GAS (in the set of positive solutions) for all values of $n \in(1, \infty)$. In fact, for $n \in(1,5]$ we have

$$
\rho_{n} \mathcal{B} \leq \rho_{n} \overline{\mathcal{B}}=\rho_{n}\left[0.031\left(1-\mathrm{e}^{-16}\right)+0.43 \frac{1-\mathrm{e}^{-16}}{1-\mathrm{e}^{-1}}\right]<1
$$

and the conclusion follows from (iii) of Corollary 4.2. For $n \geq 3$, $\mathfrak{M}=\mathfrak{M}_{1}<0.729<$ $\left(\frac{n-1}{n+1}\right)^{\frac{1}{n}}$; also, for any $n>1$,

$$
\frac{\sqrt{n \rho_{n} \mathfrak{M}_{1}^{n-1}}}{1+\mathfrak{M}_{1}^{n}}\left[0.031\left(1-\mathrm{e}^{-16}\right)+0.43 \frac{1-\mathrm{e}^{-16}}{1-\mathrm{e}^{-1}}\right]<1 \Rightarrow \frac{\sqrt{n \rho_{n} \mathfrak{M}^{n-1}}}{1+\mathfrak{M}^{n}} \mathcal{B}<1 .
$$

Thus, for $n \geq 3$ the conclusion follows from (ii) of Corollary 4.2.

On the other hand, from Proposition 4.3, the 1-periodic solution of $(5.13), y^{*}(t)$, is in the interval $(\mathfrak{m}, \mathfrak{M})=\left(\frac{0.268}{1+0.729^{n}}, 0.729\right)$. For example, if we choose $n=3$, we obtain that $y^{*}(t) \in(0.193,0.729)$ for all $t \geq 0$; however, from numerical simulations (Figure 2 ) we expect that $y^{*}(t)$ oscillates in the interval $(0.38,0.50)$.

We emphasize that the above conclusions could not be derived from previous works. In fact, for e.g. all $n \geq 2.7$, the results in [18] do not guarantee that the positive 1-periodic solution attracts all positive solutions: in this case, condition (5.9) reads as

$$
\mathcal{C}_{n}:=\frac{0.461(n-1)^{\frac{n-1}{n}}}{1-\mathrm{e}^{-1}} \leq 1
$$

Since one can show that $\mathcal{C}_{n}$ increases for $n \geq 2$ and $\mathcal{C}_{2.7}>1.01$, we conclude that the criterion given by Liu et al. in [18] cannot be applied for $n \geq 2.7$.

We now set $\eta_{1}=1.1, \eta_{2}=0.03$ and $\eta_{3}=0.001$ in equation (5.13). For this situation, we assert that the positive 1-periodic solution of (5.13) attracts all positive solutions for any $n \in(1,3]$. First, for $n \in[1.04,3]$ one can show that

$$
\rho_{n} \mathcal{B} \leq \rho_{n} \overline{\mathcal{B}}=\rho_{n}\left(1.101\left(1-\mathrm{e}^{-16}\right)+0.03 \frac{1-\mathrm{e}^{-16}}{1-\mathrm{e}^{-1}}\right)<1
$$




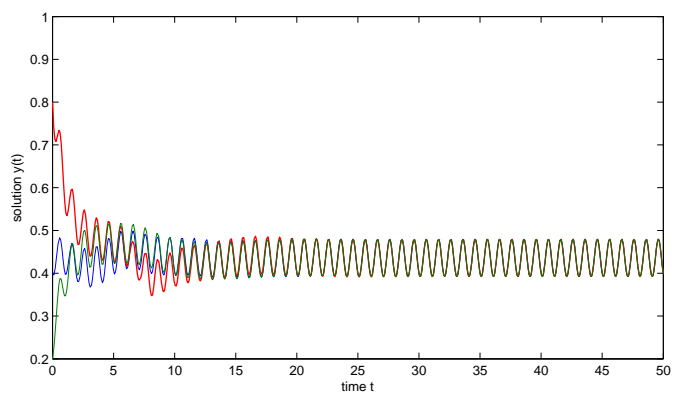

Figure 2: Numerical simulation of three solutions of (5.13) where $\eta_{1}=0.03, \eta_{2}=0.43$, $\eta_{3}=0.001$, and $n=3$, with initial condition $\varphi(\theta)=0.4\left(\frac{1}{2} \sin (\theta)+1\right), \varphi(\theta)=0.8 \mathrm{e}^{\theta}$, and $\varphi(\theta)=0.2 \mathrm{e}^{\theta}$, for $\theta \in[-16,0]$, respectively.

and the conclusion follows from condition (iii) of Corollary 4.2. But, for $n \in(1,1.04]$, we get $\rho_{n} \mathcal{B} \geq \rho_{n} \underline{\mathcal{B}}=\rho_{n}\left(1.101\left(1-\mathrm{e}^{-16}\right)+0.03 \frac{1-\mathrm{e}^{-16}}{\mathrm{e}-1}\right)>1$ and condition (iii) does not hold. However, for $n \in(1,1.04]$, we obtain

$$
\begin{aligned}
\mathfrak{m} & =\frac{1}{1+\mathfrak{M}^{n}} \max \left\{\frac{1.131}{\mathrm{e}-1}, c_{1}\right\} \\
& \geq \frac{1}{1+\mathfrak{M}^{n}} \max \left\{\frac{1.131}{\mathrm{e}-1}, 1.113\right\}=\frac{1.131}{1+c_{2}^{n}}>\frac{1.131}{1+1.167^{n}}>\left(\frac{n-1}{n+1}\right)^{\frac{1}{n}} .
\end{aligned}
$$

Recalling that $s \mapsto \frac{\sqrt{s^{n-1}}}{1+s^{n}}$ is decreasing on $\left[\left(\frac{n-1}{n+1}\right)^{\frac{1}{n}}, \infty\right)$ for all $n>1$ and $\underline{\mathfrak{m}}:=\frac{1.131}{1+1.167^{n}}$, we have

$$
\frac{\sqrt{n \rho_{n} \mathfrak{m}^{n-1}}}{1+\mathfrak{m}^{n}} \mathcal{B} \leq \frac{\sqrt{n \rho_{n} \underline{\mathfrak{m}}^{n-1}}}{1+\underline{\mathfrak{m}}^{n}} \overline{\mathcal{B}}=\frac{\sqrt{n \rho_{n} \underline{\mathfrak{m}}^{n-1}}}{1+\underline{\mathfrak{m}}^{n}}\left(1.101+\frac{0.03}{1-\mathrm{e}^{-1}}\right)\left(1-\mathrm{e}^{-16}\right)<0.72<1 .
$$

Thus the conclusion follows from condition (i) of Corollary 4.2.

Finally, we remark that simple calculations show that for these values of $\eta_{1}, \eta_{2}, \eta_{3}$ condition (5.9) is never satisfied for any $n>1$, therefore the criterion in [18] can not be invoked.

\section{Conclusions}

Blood contributes to maintaining homeostasis: a relatively steady state of internal physical and chemical conditions, controlled by living systems through a self-regulating process, in spite of the necessary adjustments for survival. This process involves negative feedback loops, to face changes, and is crucial to sustain life. Mathematically, homeostasis can be translated as the stability of some equilibrium or periodic state.

For Mackey-Glass equations (1.3), (1.4), as well as for autonomous generalizations of such models, there is an extensive number of works on the existence and stability of a positive equilibrium. For periodic versions, an a priori goal is to find sufficient conditions for the existence and global attractivity of a positive periodic solution. Although there are some recent contributions, the literature on this subject is still very scarce.

Here, we study the impulsive periodic hematopoiesis model (1.2), where linear impulses were introduced - which is in accordance with drug administration or radiation in the treatment of hematological diseases. Under sufficient conditions that ensure its existence, we give 


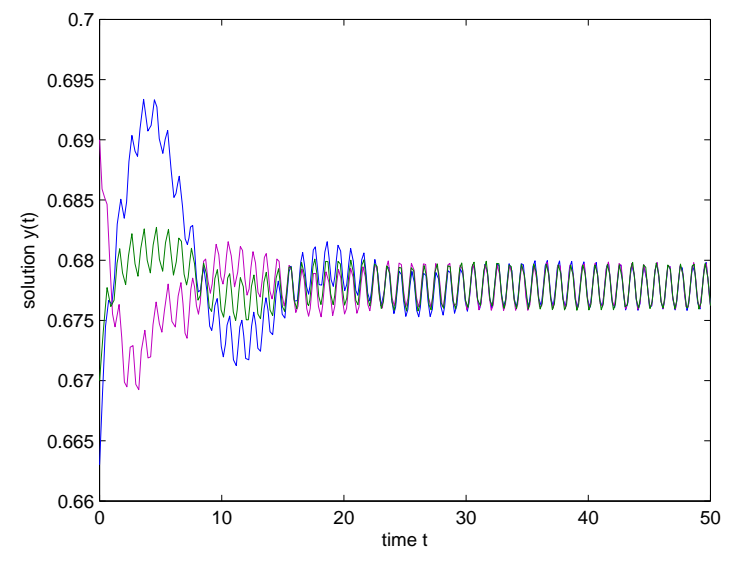

Figure 3: Numerical simulation of three solutions of (5.13) where $\eta_{1}=1.1, \eta_{2}=0.03$, $\eta_{3}=0.001$ and $n=1.03$, with initial condition $\varphi(\theta)=0.67, \varphi(\theta)=0.65(1+0.02 \cos (\theta))$, and $\varphi(\theta)=0.69(1+0.02 \sin (\theta))$, for $\theta \in[-16,0]$, respectively.

new criteria for the global asymptotic stability of a positive periodic solution of (1.2). We hope that these results can be used in applications, namely in the use of impulses to treat hematopoiesis disorders. The main results are given in Theorems 4.1 and 4.3, respectively for the cases $n \in(0,1]$ and $n \in(1, \infty)$, although the criteria in Corollaries 4.1 and 4.2 are easier to apply.

In Section 5, we study several models, with and without impulses, and conclude that our achievements enhance the few previous criteria set up by other authors.

Linear impulses seem to fit well with the clinical procedure. The constraints imposed here on the linear impulses are weaker than the requirements often found in the literature, nevertheless we would like to generalize our techniques to DDEs with nonlinear impulses.

We note that the study of the global attractivity of a periodic solution for (1.2) relies on the results in Section 3, where a broad family of scalar impulsive DDEs (1.1) was considered and sufficient conditions for the GAS of its zero solution established. It is apparent that the technique employed here can be used for almost periodic models (1.2), rather than periodic, provided that a positive almost periodic solution exists: in fact, the arguments in Subsections 4.2 and 4.3 do not make use of the periodicity of (1.2); of course, in this case one should replace $\sup _{t \in[0, \omega]}$ by $\sup _{t \geq 0}$ in the definition of $\mathcal{A}, \mathcal{B}$ in Corollaries 4.1 and 4.2 .

\section{Acknowledgements}

This work was partially supported by Fundação para a Ciência e a Tecnologia (Portugal) under project UID/MAT/04561/2019 (Teresa Faria) and project UID/MAT/00013/2013 (José J. Oliveira). The authors express their gratitude to the referees and editors, for their valuable comments and suggestions.

\section{References}

[1] T. Faria, J.J. Oliveira, Existence of positive periodic solutions for scalar delay differential equations with and without impulses. J. Dyn. Differ. Equ. (to appear). 
https://doi.org/10.1007/s10884-017-9616-0

[2] M.C. Mackey, L. Glass, Oscillation and chaos in physiological control system, Science 197 (1977) 287-289. https://doi.org/10.1126/science.267326

[3] C. Foley, M.C. Mackey, Dynamic hematological disease: a review, J. Math. Biol. 58 (2009), 285-322. https://doi.org/10.1007/s00285-008-0165-3

[4] J.M. Mahaffy, J. Bélair, M.C. Mackey, Hematopoietic model with moving boundary condition and state dependent delay, J. Theor. Biol. 190 (1998), 135-146. https://doi.org/10.1006/jtbi.1997.0537

[5] M.C. Mackey, Unified hypothesis of the origin of aplastic anaemia and periodic hematopoiesis, Blood 51 (1978), 941-956.

[6] M. C. Mackey, Dynamic hematological disorders of stem cell origin, in G. Vassileva-Popova and E. V. Jensen (Eds), Biophysical and Biochemical Information Transfer in Recognition, Plenum Press, New York, 1979, pp. 373-409.

[7] K. Gopalsamy, M.R.S. Kulenovic, G. Ladas, Oscillation and global attractivity in models of hematopoiesis, J. Dyn. Differ. Equ. 2 (1990), 117-132. https://doi.org/10.1007/BF02514684

[8] K. Gopalsamy, S. Trofimchuk, N. Bantsur, A note on global attractivity in models of hematopoiesis, Ukrainian Math. J. 50 (1998), 3-12.

[9] I. Győri, G. Ladas, Oscillation Theory of Delay Differential Equations with Applications, Clarendon, Oxford, 1991.

[10] G. Karakostas, Ch.G. Philos, Y.G. Sficas, Stable steady state of some population models, J. Dyn. Differ. Equ. 4 (1992) 161-190. https://doi.org/10.1007/BF01048159

[11] E. Liz, M. Pinto, M. Tkachenko, V. Trofimchuk, A global stability criterion for a family of delayed population models, Quart. Appl. Math. 63 (2005), 56-70. https://doi.org/10.1090/S0033569X-05-00951-3

[12] J. Bélair, M. C. Mackey, J. M. Mahaffy, Age-structured and two-delay models for erythropoiesis, Math. Biosci. 128 (1995), 317-346. https://doi.org/10.1016/0025-5564(94)00078-E

[13] C. Haurie, D.C. Dale, M.C. Mackey, Cyclical neutropenia and o ther periodic hematological diseases: A review of mechanisms and mathematical models, Blood 92 (1998), 2629-2640.

[14] M. Adimy, F. Crauste, S. Ruan, Modelling hematopoiesis mediated by growth factors with applications to periodic hematological diseases, Bull. Math. Biol. 68 (2006), 2321-2351. https://doi.org/10.1007/s11538-006-9121-9

[15] G.P. Langlois, M. Craig, A.R. Humphries et al., Normal and pathological dynamics of platelets in humans, J. Math. Biol. 75 (2017), 1411-1462. https://doi.org/10.1007/s00285-017-1125-6

[16] Y. Yan, J. Sugie, Existence regions of positive periodic solutions for a discrete hematopoiesis model with unimodal production functions. Appl. Math. Model. 68 (2019), 152-168. https://doi.org/10.1016/j.apm.2018.11.003

[17] P. Amster, L. Idels, Periodic solutions in general scalar non-autonomous models with delays, Nonlinear Differential Equations Appl. 20 (2013), 1577-1596. https://doi.org/10.1007/s00030013-0222-5

[18] G. Liu, J. Yan, F. Zhang, Existence and global attractivity of unique positive periodic solution for a model of hematopoiesis, J. Math. Anal. Appl. 334 (2007), 157-171. https://doi.org/10.1016/j.jmaa.2006.12.015 
[19] S.H. Saker, Oscillation and global attractivity in a periodic delay hematopoiesis model, J. Appl. Math. Comput. 13 (2003), 287-300. https://doi.org/10.1007/BF02936093

[20] X. Wang, Z. Li, Dynamics for a class of general hematopoiesis model with periodic coefficients. Appl. Math. Comput. 186 (2007), 460-468. Erratum: Appl. Math. Comput. 2006 (2008), 473476. https://doi.org/10.1016/j.amc.2006.07.109

[21] L. Berezansky, E. Braverman, L. Idels, Mackey-Glass model of hematopoiesis with monotone feedback revisited, Appl. Math. Comput. 219 (2013), 4892-4907. https://doi.org/10.1016/j.amc.2012.10.052

[22] L. Berezansky, E. Braverman, L. Idels, Mackey-Glass model of hematopoiesis with nonmonotone feedback: stability, oscillation and control, Appl. Math. Comput. 219 (2013), 62686283. https://doi.org/10.1016/j.amc.2012.12.043

[23] X. Liu, Y. Takeuchi, Periodicity and global dynamics of an impulsive delay Lasota-Wazewska model, J. Math. Anal. Appl. 327 (2007), 326-341. https://doi.org/10.1016/j.jmaa.2006.04.026

[24] S.H. Saker, J.O. Alzabut, On the impulsive delay hematopoiesis model with periodic coefficients, Rocky Mountain J. Math 39 (2009), 1657-1688. https://doi:10.1216/RMJ-2009-39-5-1657

[25] A.M. Samoilenko, N.A. Perestyuk, Impulsive Differential Equations, World Scientific, Singapore, 1995.

[26] L. Berezansky, E. Braverman, A note on stability of Mackey-Glass equations with two delays, J. Math. Anal. Appl. 450 (2017), 1208-1228. https://doi.org/10.1016/j.jmaa.2017.01.050

[27] H.S. Ding, Q.-L. Liu, J.J. Nieto, Existence of positive almost periodic solutions to a class of hematopoiesis model, Appl. Math. Model. 40 (2016), 3289-3297. https://doi.org/10.1016/j.apm.2015.10.020

[28] Z. Yao, New results on existence and exponential stability of the unique positive almost periodic solution for Hematopoiesis model, Appl. Math. Modelling, 9 (2015), 7113-7123. https://doi.org/10.1016/j.apm.2015.03.003

[29] Z. Yao, Almost periodicity of impulsive hematopoiesis model with infinite delay, J. Nonlinear Sci. Appl. 8 (2015), 856-865. https://doi.org/10.22436/jnsa.008.05.35

[30] J.O. Alzabut, J.J. Nieto, G. Tr. Stamov, Existence and exponential stability of positive almost periodic solutions for a model of hematopoiesis, Bound. Value Probl. 2009 (2019), Art. ID 127510, 10 pp. https://doi.org/10.1155/2009/127510

[31] T. Faria, J.J. Oliveira, A note on stability of impulsive scalar delay differential equations, Electron. J. Qual. Theory Differ. Equ. 2016, Paper No. 69, 14 pp. https://doi.org/10.14232/ejqtde.2016.1.69

[32] T. Faria, J.J. Oliveira, On stability for impulsive delay differential equations and applications to a periodic Lasota-Wazewska model. Discrete Contin. Dyn. Syst. Ser. B 21 (2016), 2451-2472. https://doi.org/10.3934/dcdsb.2016055

[33] T. Faria, J.J. Oliveira, A note on global attractivity of the periodic solution for a model of hematopoiesis, Appl. Math. Lett. 94 (2019), 1-7. https://doi.org/10.1016/j.aml.2019.02.009

[34] T. Faria, M.C. Gadotti and J.J. Oliveira, Stability results for impulsive functional differential equations with infinite delay. Nonlinear Anal. 75 (2012) 6570-6587. https://doi.org/10.1016/j.na.2012.07.030 
[35] J. Yan, Stability for impulsive delay differential equations. Nonlinear Anal. 63 (2005), 66-80. https://doi.org/10.1016/j.na.2005.05.001

[36] J.A. Yorke, Asymptotic stability for one dimensional delay-differential equations. J. Differential Equations 7 (1970), 189-202. https://doi.org/10.1016/0022-0396(70)90132-4 